\title{
Computation of eigenmodes on a compact hyperbolic 3-space
}

\author{
KAIKI TARO INOUE \\ Yukawa Institute for Theoretical Physics \\ Kyoto University, Kyoto 606-8502, Japan
}

\begin{abstract}
Measurements of cosmic microwave background (CMB) anisotropy are ideal experiments for discovering the non-trivial global topology of the universe. To evaluate the CMB anisotropy in multiply-connected compact cosmological models, one needs to compute the eigenmodes of the Laplace-Beltrami operator. Using the direct boundary element method, we numerically obtain the low-lying eigenmodes on a compact hyperbolic 3-space called the Thurston manifold which is the second smallest in the known compact hyperbolic 3-manifolds. The computed eigenmodes are expanded in terms of eigenmodes on the unit three-dimensional pseudosphere. We numerically find that the expansion coefficients behave as Gaussian pseudorandom numbers for low-lying eigenmodes. The observed gaussianity in the CMB fluctuations can partially be attributed to the Gaussian pseudo-randomness of the expansion coefficients assuming that the Gaussian pseudo-randomness is the universal property of the compact hyperbolic spaces.
\end{abstract}




\section{Introduction}

In recent years, there has been a great interest in properties of CMB anisotropy in multiply-connected cosmological models [1, 2, 3, 4]. Most of these studies deal with flat models or non-compact hyperbolic models for which the eigenmodes are known explicitly. Since no closed analytic expression of eigenmodes is known for compact hyperbolic $(\mathrm{CH})$ models, so far, analysis of the CMB anisotropy in $\mathrm{CH}$ models has been considered to be quite difficult although they have interesting properties which are strikingly different from that of multiply-connected flat models. For instance, in low $\Omega_{o}$ adiabatic models, the large-angular fluctuations can be produced at periods after the last scattering as the curvature perturbations decay in the curvature dominant era. Therefore, the argument of the suppression of the large-angular fluctuations due to the "mode-cutoff" in the multiply-connected flat models cannot simply be applicable to the multiply-connected hyperbolic models.

Because the effect of the multiply-connectedness becomes significant as the volume of the space becomes small, it is very important to study whether the"small" universe scenario is plausible. For instance, the Weeks manifold and the Thurston manifold have volume $\sim R^{3}$ where $R$ denotes the curvature radius, and they are the smallest and the second smallest compact hyperbolic manifolds, respectively. For a technical reason, we study the properties of eigenmodes on the Thurston manifold. Since each type(scalar, vector and tensor) of perturbations evolves independently on the locally homogeneous and isotropic FRW background space, in $k$-space, the perturbations are given by the eigenmodes and the time evolution of the perturbations on the locally homogeneous and isotropic FRW space. The periodic boundary conditions on the eigenmodes drastically change the nature of the CMB fluctuations on the topological identification scale while on smaller scale they asymptotically converge to that in the standard locally and globally homogeneous and isotropic FRW background space. Thus computation of eigenmodes are very important in understanding the properties of CMB fluctuations 円.

Computing the eigenmodes of the Laplace-Beltrami operator in $\mathrm{CH}$ spaces

\footnotetext{
${ }^{1}$ It should be noted that the computation of eigenmodes is also essential in the framework of spectral geometry [5].
} 
(manifolds) is equivalent to solving the Helmholtz equation with appropriate periodic boundary conditions in the universal covering space. A number of numerical methods have been used for solving the Helmholtz equation such as the finite element methods and the finite difference methods [6, 7, 8].

A numerical method called the "direct boundary element method" (DBEM) has been used by Aurich and Steiner for finding out the eigenmodes of the LaplaceBeltrami operator in a two-dimensional compact multiply-connected space for studying the statistical properties of the eigenmodes in highly-excited states or equivalently the semi-classical wavefunctions [9]. We find that this pioneering work for "quantum chaology" , the study of the imprints of classical chaos in the quantum mechanical counterparts is very useful for the study of the CMB anisotropy in $\mathrm{CH}$ cosmological models as well. The advantage of the DBEM is that it reduces the dimensionality of the problem by one which leads to economy in the numerical task. Since one needs to discretize only the boundary, generation of meshes is much easier than the other methods. Furthermore, as we shall see later, the DBEM is suitable for expanding the eigenfunctions that are continued onto the whole Poincaré ball by the periodic boundary conditions in terms of eigenfunctions on the pseudosphere ${ }^{2}$. In order to compute a set of expansion coefficients, one needs to compute the values of the corresponding eigenfunction on a hyperbolic sphere with appropriate radius. If one does not care about the normalization of the eigenfunctions, unlike the FEM, the computation of the eigenfunctions on the whole fundamental domain is not necessary. In the DBEM, computation of the eigenfunction at an arbitrary point either inside or outside of the fundamental domain can be done by using the values of the eigenfunction and the normal derivatives on the boundary.

As the classical dynamical systems in $\mathrm{CH}$ spaces are strongly chaotic, one can naturally assume that the imprint of the classical chaos is hidden in the corresponding quantum systems in some way. It has been found that the expansion coefficients with a certain basis behave as if they are random Gaussian numbers in some classically chaotic systems ([9, 10), which is consistent with the prediction of random-matrix theory $([11])$. Since the CMB temperature fluctuations in

\footnotetext{
${ }^{2} \mathrm{~A}$ set of the continued eigenfunctions is a subset of all eigenfunctions on the universal covering space.
} 
$\mathrm{CH}$ spaces are written in terms of expansion coefficients and the eigenfunctions on the universal covering space plus initial fluctuations, if the random behavior of the expansion coefficients is confirmed, the origin of the random gaussianity in the CMB temperature fluctuations can be partially explained in terms of the geometric property of the universe.

In this paper, we introduce the DBEM for solving the Helmholtz equation. Then we apply the DBEM for computing the low-lying eigenmodes of the LaplaceBeltrami operator in the Thurston manifold. The computed eigenfunctions are naturally continued onto the whole Poincaré ball because of the periodic boundary conditions and are expanded in terms of eigenmodes on the simply-connected pseudosphere. Statistical properties of the expansion coefficients are examined, since they are key factors in understanding of the CMB anisotropy in $\mathrm{CH}$ models.

\section{The direct boundary element method (DBEM)}

The boundary element methods (BEM) use free Green's function as the weighted function, and the Helmholtz equation is rewritten as an integral equation defined on the boundary using Green's theorem. Discretization of the boundary integral equation yields a system of linear equations. Since one needs the discretiztion on only the boundary, BEM reduces the dimensionality of the problem by one which leads to economy in the numerical task. To locate an eigenvalue, the DBEM 5 requires one to compute many determinants of the corresponding boundary matrices which are dependent on the wavenumber $k$.

Firstly, let us consider the Helmholtz equation with certain boundary conditions,

$$
\left(\nabla^{2}+k^{2}\right) u(\mathbf{x})=0
$$

which is defined on a bounded M-dimensional connected and simply-connected domain $\Omega$ which is a subspace of a M-dimensional Riemannian manifold $\mathcal{M}$ and the boundary $\partial \Omega$ is piecewise smooth. $\nabla^{2} \equiv \nabla^{i} \nabla_{i},(i=1,2, \cdots, M)$, and $\nabla_{i}$ is the

\footnotetext{
${ }^{3}$ The DBEM uses only boundary points in evaluating the integrand in Eq.(5). The indirect methods use internal points in evaluating the integrand in Eq.(5) as well as the boundary points.
} 
covariant derivative operator defined on $\mathcal{M}$. A function $u$ in Sobolev space $H^{2}(\Omega)$ is the solution of the Helmholtz equation if and only if

$$
\mathcal{R}[u(\mathbf{x}), v(\mathbf{x})] \equiv\left\langle\left(\nabla^{2}+k^{2}\right) u(\mathbf{x}), v(\mathbf{x})\right\rangle=0,
$$

where $v$ is an arbitrary function in Sobolev space $H^{1}(\Omega)$ called weighted function and \langle\rangle is defined as

$$
\langle a, b\rangle \equiv \int_{\Omega} a b \sqrt{g} d V
$$

Next, we put $u(\mathbf{x})$ into the form

$$
u=\sum_{j=1}^{M} u_{j} \phi_{j}
$$

where $\phi_{j}$ 's are linearly independent square-integrable functions. Numerical methods such as the finite element methods try to minimize the residue function $\mathcal{R}$ for a fixed weighted function $v(\mathbf{x})$ by changing the coefficients $u_{j}$. In these methods, one must resort to the variational principle to find the $u_{j}$ 's which minimize $\mathcal{R}$.

Now we formulate the DBEM which is a version of BEMs. Here we search $u(\mathbf{x})$ 's for the space $C^{1}(\bar{\Omega}) \cap C^{2}(\Omega) \cap L^{2}(\Omega)$. First, we slightly modify Eq.(2) using the Green's theorem

$$
\int_{\Omega}\left(\nabla^{2} u\right) v \sqrt{g} d V-\int_{\Omega}\left(\nabla^{2} v\right) u \sqrt{g} d V=\int_{\partial \Omega}\left(\nabla_{i} u\right) v \sqrt{g} d S^{i}-\int_{\partial \Omega}\left(\nabla_{i} v\right) u \sqrt{g} d S^{i}
$$

where $g \equiv \operatorname{det}\left\{g_{i j}\right\}$ and $d V \equiv d x_{1} \ldots d x_{M}$; the surface element $d S^{i}$ is given by

$$
\begin{aligned}
d S_{i} & \equiv \frac{1}{M !} \epsilon_{i j_{1} \cdots j_{M}} d S^{j_{1} \cdots j_{M}} \\
d S^{j_{1} \ldots j_{M}} & \equiv\left|\begin{array}{cccc}
d x^{(1) j_{1}} & d x^{(2) j_{1}} & \ldots & d x^{(M) j_{1}} \\
d x^{(1) j_{2}} & d x^{(2) j_{2}} & \ldots & d x^{(M) j_{2}} \\
\vdots & \vdots & \ddots & \vdots \\
d x^{(1) j_{M}} & d x^{(2) j_{M}} & \ldots & d x^{(M) j_{M}}
\end{array}\right|
\end{aligned}
$$

where $\epsilon_{j_{1} \cdots j_{M+1}}$ denotes the M+1-dimensional Levi-Civita tensor. Then Eq.(2) becomes

$$
\int_{\Omega}\left(\nabla^{2} v+k^{2} v\right) u \sqrt{g} d V+\int_{\partial \Omega}\left(\nabla_{i} u\right) v \sqrt{g} d S^{i}-\int_{\partial \Omega}\left(\nabla_{i} v\right) u \sqrt{g} d S^{i}=0 .
$$


As the weighted function v, we choose the fundamental solution $G_{E}(\mathbf{x}, \mathbf{y})$ which satisfies

$$
\left(\nabla^{2}+E\right) G_{E}(\mathbf{x}, \mathbf{y})=\delta_{D}(\mathbf{x}-\mathbf{y}),
$$

where $E \equiv k^{2}$, and $\delta_{D}(\mathbf{x}-\mathbf{y})$ is Dirac's delta function. $G_{E}(\mathbf{x}, \mathbf{y})$ is also known as the free Green's function whose boundary condition is given as

$$
\lim _{d(\mathbf{x}, \mathbf{y}) \rightarrow \infty} G_{E}(\mathbf{x}, \mathbf{y})=0,
$$

where $d(\mathbf{x}, \mathbf{y})$ is the geodesic distance between $\mathbf{x}$ and $\mathbf{y}$. Let $\mathbf{y}$ be an internal point of $\Omega$. Then we obtain from Eq.(7) and Eq.(8),

$$
u(\mathbf{y})+\int_{\partial \Omega} G_{E}(\mathbf{x}, \mathbf{y}) \nabla_{i} u \sqrt{g} d S^{i}-\int_{\partial \Omega}\left(\nabla_{i} G_{E}(\mathbf{x}, \mathbf{y})\right) u \sqrt{g} d S^{i}=0 .
$$

Thus the values of eigenfunctions at internal points can be computed using only the boundary integral. If $\mathbf{y} \in \partial \Omega$, we have to evaluate the limit of the boundary integral terms as $G_{E}(\mathbf{x}, \mathbf{y})$ becomes divergent at $\mathbf{x}=\mathbf{y}$ (see appendix A). The boundary integral equation is finally written as

$$
\frac{1}{2} u(\mathbf{y})+\int_{\partial \Omega} G_{E}(\mathbf{x}, \mathbf{y}) \nabla_{i} u \sqrt{g} d S^{i}-\int_{\partial \Omega}\left(\nabla_{i} G_{E}(\mathbf{x}, \mathbf{y})\right) u \sqrt{g} d S^{i}=0,
$$

or in another form,

$$
\frac{1}{2} u(\mathbf{y})+\int_{\partial \Omega} G_{E}(\mathbf{x}, \mathbf{y}) \frac{\partial u}{\partial x^{i}} n^{i} \sqrt{g} d S-\int_{\partial \Omega} \frac{\partial G_{E}(\mathbf{x}, \mathbf{y})}{\partial x^{i}} n^{i} u \sqrt{g} d S=0
$$

where $n^{i} \equiv d S^{i} / d S$ and $d S \equiv \sqrt{d S^{i} d S_{i}}$. Note that we assumed that the boundary surface at $\mathbf{y}$ is sufficiently smooth. If the boundary is not smooth, one must calculate the internal solid angle at $\mathbf{y}$ (see appendix A). Another approach is to rewrite Eq.(10) in a regularized form [13]. We see from Eq.(11) or Eq.(12) that the approximated solutions can be obtained without resorting to the variational principle. Since it is virtually impossible to solve Eq.(12) analytically, we discretize it using boundary elements. Let the number of the elements be $\mathrm{N}$. We approximate $u$ by some low-order polynomials (shape function) on each element as $u=c_{1}+$ $c_{2} \eta+c_{3} \xi$ where $\eta$ and $\xi$ denote the coordinates on the corresponding standard element 1 .

\footnotetext{
${ }^{4}$ It can be proved that the approximated polynomial solutions converge to $u(\mathbf{x})$ as the number of boundary elements becomes large [14, 15].
} 
Then we have the following equation:

$$
[H]\{u\}=[G]\{q\}, \quad q \equiv \frac{\partial u}{\partial n},
$$

where $\{u\}$ and $\{q\}$ are N-dimensional vectors which consist of the boundary values of an eigenfunction and its normal derivatives, respectively. $[\mathrm{H}]$ and $[\mathrm{G}]$ are $\mathrm{N} \times \mathrm{N}$ - dimensional coefficient matrices which are obtained from integration of the fundamental solution $G_{E}(\mathbf{x}, \mathbf{y})$ and its normal derivatives multiplied by $u_{i}$ and $q_{i}$, respectively. The explicit form of $[\mathrm{H}]$ and $[\mathrm{G}]$ for constant elements are given in section 4. Note that the elements in $[\mathrm{H}]$ and $[\mathrm{G}]$ include $k$ implicitly. Because Eq.(13) includes both $u$ and $q$, the boundary element method can naturally incorporate the periodic boundary conditions:

$$
u(\mathbf{x})=u\left(g_{i}(\mathbf{x})\right), \quad q(\mathbf{x})=-q\left(g_{i}(\mathbf{x})\right), \quad \text { on } \quad \partial \Omega,
$$

where $g_{i}$ 's are the face-to-face identification maps defined on the boundary(see appendix B). The boundary conditions constrain the number of unknown constants to N. Application of the boundary condition (14) to Eq.(13) and permutation of the columns of the components yields

$$
[A]\{x\}=0,
$$

where $\mathrm{N} \times \mathrm{N}$ - dimensional matrix $A$ is constructed from $G_{i j}$ and $H_{i j}$ and N-dimensional vector $x$ is constructed from $u_{i}{ }^{\prime}$ s and $q_{i}{ }^{\prime}$ s. For the presence of the non-trivial solution, the following relation must hold,

$$
\operatorname{det}[A]=0 .
$$

Thus the eigenvalues of the Laplace-Beltrami operator acting on the space $C^{1}(\bar{\Omega}) \cap$ $C^{2}(\Omega) \cap L^{2}(\Omega)$ are obtained by searching for $k$ 's which satisfy Eq.(16). 


\section{Computation of low-lying eigenmodes}

In this section, we apply the DBEM for computing the low-lying eigenmodes on the Thurston manifold $Q_{2}$. We have chosen $Q_{2}$ for a technical reason that the fundamental domain of $Q_{2}$ is much simpler than that of the Weeks manifold $Q_{1}$ (generation of meshes is much simpler). See appendix B for understanding the basic aspects of three-dimensional hyperbolic geometry.

The Helmholtz equation in the Poincaré coordinates is written as

$$
\frac{1}{4}\left(1-|\mathbf{x}|^{2}\right)^{2}\left[\Delta_{E}+\frac{2}{1-|\mathbf{x}|^{2}} \mathbf{x} \cdot \nabla_{E}\right] u+k^{2} u=0
$$

where $\Delta_{E}$ and $\nabla_{E}$ are the Laplacian and the gradient on the corresponding threedimensional Euclidean space, respectively. Note that we have set the curvature radius $R=1$ without loss of generality. By using the DBEM, the Helmholtz equation (17) is converted to an integral representation on the boundary. Here Eq.(12) can be written in terms of Euclidean quantities as

$$
\frac{1}{2} u(\mathbf{y})+\int_{\partial \Omega} G_{k}(\mathbf{x}, \mathbf{y}) \frac{\partial u}{\partial x^{i}} n_{E}^{i} d S-\int_{\partial \Omega} \frac{\partial G_{k}(\mathbf{x}, \mathbf{y})}{\partial x^{i}} u n_{E}^{i} d S=0
$$

where $d S=2\left(1-|\mathbf{x}|^{2}\right)^{-1} d S_{E}$. The fundamental solution is given as [16, 17]

$$
G_{k}(\mathbf{x}, \mathbf{y})=-\frac{1}{4 \pi} \frac{\left(\sigma+\sqrt{\sigma^{2}-1}\right)^{-s}}{\sqrt{\sigma^{2}-1}}, \quad-\frac{\pi}{2}<\arg s \leq \frac{\pi}{2},
$$

where $s=\sqrt{1-k^{2}}$ and $\sigma=\cosh d(\mathbf{x}, \mathbf{y})$. Then Eq. (18) is discretized on the boundary elements $\Gamma_{J}$ as

$$
\frac{1}{2} u\left(\mathbf{x}_{I}\right)+\sum_{J=1}^{N}\left[\int_{\Gamma_{J}} G_{k}\left(\mathbf{x}_{I}, \mathbf{y}_{J}\right) \frac{\partial u\left(\mathbf{y}_{J}\right)}{\partial n} d S-\int_{\Gamma_{J}} \frac{\partial G_{k}\left(\mathbf{x}_{I}, \mathbf{y}_{J}\right)}{\partial n} u\left(\mathbf{y}_{J}\right) d S\right]=0
$$

where $\mathrm{N}$ denotes the number of the boundary elements. An example of $N=1168$ elements on the boundary of the fundamental domain in the Poincaré coordinates is shown in figure 1. These elements are firstly generated in Klein coordinates in which the mesh-generation is convenient. The maximum length of the edge $\Delta l$ in these elements is 0.14. The condition that the corresponding de Broglie wavelength $2 \pi / k$ is longer than the four times of the interval of the boundary elements yields 


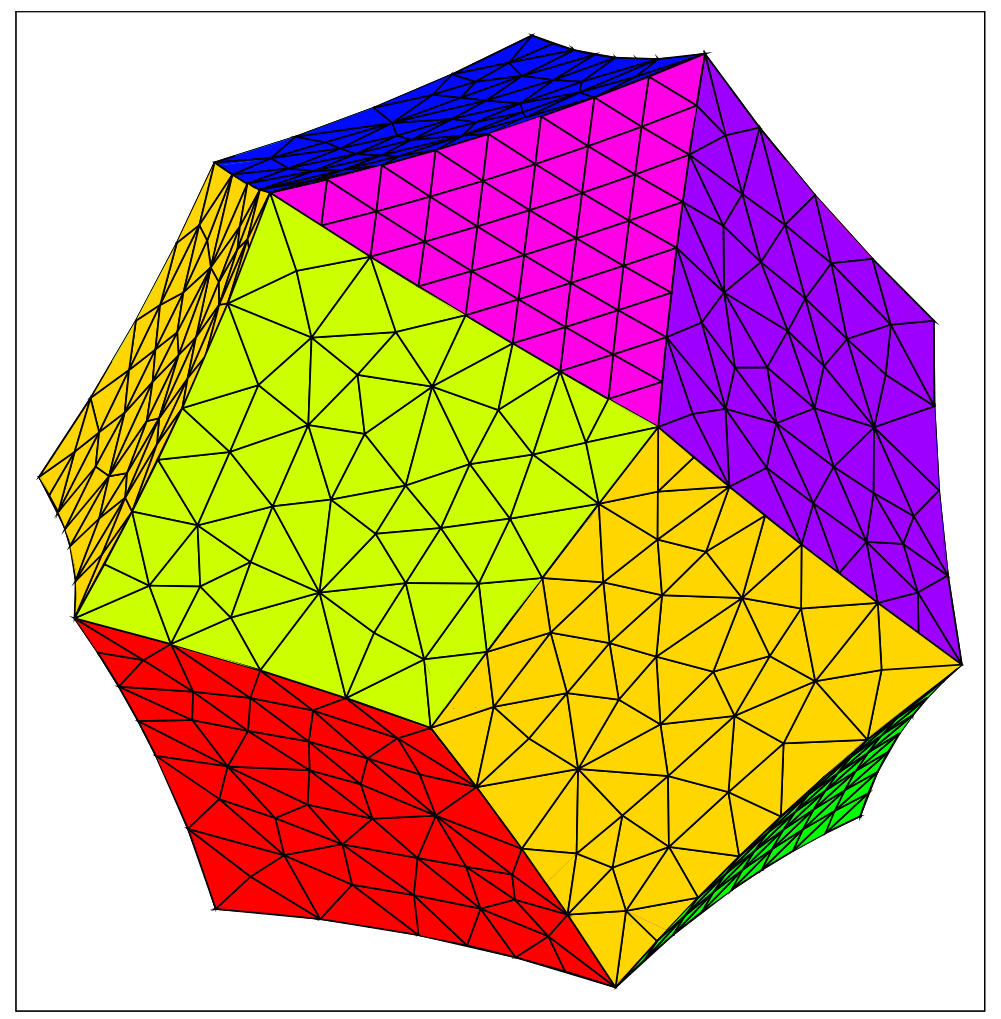

Figure 1: 1168 boundary elements 
a rough estimate of the validity condition of the calculation as $k<11$. On each $\Gamma_{J}$, $\mathrm{u}$ and $\mathrm{q} \equiv \partial u / \partial n$ are approximated by low order polynomials. For simplicity, we use constant elements:

$$
u\left(\mathbf{x}_{J}\right)=u^{J}=\text { Const. }, \quad q\left(\mathbf{x}_{J}\right)=q^{J}=\text { Const. }, \quad \text { on } \Gamma_{J} .
$$

Substituting Eq.(21) into Eq.(20), we obtain

$$
\begin{aligned}
\sum_{J=1}^{N} H_{I J} u^{J} & =\sum_{J=1}^{N} G_{I J} q^{J}, \\
H_{I J} & = \begin{cases}\tilde{H}_{I J} & I \neq J \\
\tilde{H}_{I J}-\frac{1}{2} & I=J\end{cases}
\end{aligned}
$$

where

$$
\tilde{H}_{I J} \equiv \int_{\Gamma_{J}} \frac{\partial G_{k}}{\partial n}\left(\mathbf{x}_{I}, \mathbf{y}_{J}\right) d S\left(\mathbf{y}_{J}\right), \quad G_{I J} \equiv \int_{\Gamma_{J}} G_{k}\left(\mathbf{x}_{I}, \mathbf{y}_{J}\right) d S\left(\mathbf{y}_{J}\right)
$$

The singular integration must be carried out for I-I components as the fundamental solution diverges at $\left(\mathbf{x}_{I}=\mathbf{y}_{I}\right)$. This is not an intractable problem. Several numerical techniques have already been proposed by some authors [18, 19. We have applied Hayami's method to the evaluation of the singular integrals [19]. Introducing coordinates similar to spherical coordinates centered at $\mathbf{x}_{I}$, the singularity is canceled out by the Jacobian which makes the integral regular.

Let $g_{i}(i=1,2, \ldots, 8)$ be the generators of the discrete group $\Gamma$ which identify a boundary face $F_{i}$ with another boundary face $g_{i}\left(F_{i}\right)$ :

$$
g_{i}\left(\mathbf{x}_{i}\right)=\mathbf{x}_{i}, \quad \mathbf{x}_{i} \in F_{i}
$$

The boundary of the fundamental domain can be divided into two regions $\partial \Omega_{A}$ and $\partial \Omega_{B}$ and each of them consists of $N / 2$ boundary elements,

$$
\partial \Omega_{A}=\cup F_{i}, \quad \partial \Omega_{B}=\cup g_{i}\left(F_{i}\right), \quad i=1,2, \ldots, 8 .
$$

The periodic boundary conditions

$$
u\left(g_{i}\left(\mathbf{x}_{i}\right)\right)=u\left(\mathbf{x}_{i}\right), \quad q\left(g_{i}\left(\mathbf{x}_{i}\right)\right)=-q\left(\mathbf{x}_{i}\right), \quad i=1,2, \ldots, 8
$$


reduce the number of the independent variables to $\mathrm{N}$, i.e. for all $\mathbf{x}_{B} \in \partial \Omega_{B}$, there exist $g_{i} \in \Gamma$ and $\mathbf{x}_{A} \in \partial \Omega_{A}$ such that

$$
u\left(\mathbf{x}_{B}\right)=u\left(g_{i}\left(\mathbf{x}_{A}\right)\right)=u\left(\mathbf{x}_{A}\right), \quad q\left(\mathbf{x}_{B}\right)=-q\left(g_{i}\left(\mathbf{x}_{A}\right)\right)=-q\left(\mathbf{x}_{A}\right)
$$

Substituting the above relation into Eq.(22), we obtain

$$
\left[\begin{array}{ll}
H_{A A}+H_{A B} & -G_{A A}+G_{A B} \\
H_{B A}+H_{B B} & -G_{B A}+G_{B B}
\end{array}\right]\left\{\begin{array}{l}
u_{A} \\
q_{A}
\end{array}\right\}=0
$$

where $u_{A}=\left(u^{1}, u^{2}, \ldots u^{N / 2}\right)$ and $q_{A}=\left(q^{1}, q^{2}, \ldots q^{N / 2}\right)$ and matrices $H=\left\{H_{I J}\right\}$ and $G=\left\{G_{I J}\right\}$ are written as

$$
H=\left[\begin{array}{ll}
H_{A A} & H_{A B} \\
H_{B A} & H_{B B}
\end{array}\right], \quad G=\left[\begin{array}{ll}
G_{A A} & G_{A B} \\
G_{B A} & G_{B B}
\end{array}\right] .
$$

Eq. (27) takes the form

$$
[A(k)]\{x\}=0,
$$

where $\mathrm{N} \times \mathrm{N}$ - dimensional matrix $A$ is constructed from $G$ and $H$ and $\mathrm{N}$-dimensional vector $x$ is constructed from $u_{A}$ and $q_{A}$. For the presence of the non-trivial solution, the following relation must hold,

$$
\operatorname{det}[A(k)]=0
$$

Thus the eigenvalues of the Laplace-Beltrami operator in a $\mathrm{CH}$ space are obtained by searching for $k$ 's which satisfy Eq.(30). In practice, Eq.(30) cannot be exactly satisfied as the test function which has a locally polynomial behavior is slightly deviated from the exact eigenfunction. Instead, one must search for the local minima of $\operatorname{det}[\mathrm{A}(\mathrm{k})]$. This process needs long computation time as $A(k)$ depends on $\mathrm{k}$ implicitly. Our numerical result $(k<10)$ is shown in table 1 .

The first "excited state" which corresponds to $k=k_{1}$ is important for the understanding of $\mathrm{CMB}$ anisotropy. Our numerical result $k_{1}=5.41$ is consistent with the value 5.04 obtained from Weyl's asymptotic formula

$$
N[\nu]=\frac{\operatorname{Vol}(\mathcal{M}) \nu^{3}}{6 \pi^{2}}, \quad \nu \equiv \sqrt{k^{2}-1}, \quad \nu>>1,
$$

assuming that no degeneracy occurs. One can interpret the first excited state as the mode that has the maximum de Broglie wavelength $2 \pi / k_{1}$. Because of 


\begin{tabular}{cc}
\hline $\mathrm{k}$ & $m_{k}$ \\
\hline 5.41 & 1 \\
\hline 5.79 & 1 \\
\hline 6.81 & 1 \\
\hline 6.89 & 1 \\
\hline 7.12 & 1 \\
\hline 7.69 & 1 \\
\hline 8.30 & 1 \\
\hline 8.60 & 1 \\
\hline 8.73 & 1 \\
\hline 9.26 & 2 \\
\hline 9.76 & 1 \\
\hline 9.91 & 1 \\
\hline 9.99 & 1 \\
\hline
\end{tabular}

Table 1: Eigenvalue $\mathrm{k}$ and multiplicity $m_{k}$

the periodic boundary conditions, the de Broglie wavelength can be approximated by the "average diameter" of the fundamental domain defined as a sum of the inradius $r_{-}$and the outradius $r_{+}$? , which yields $k_{1}=4.9$ just $10 \%$ less than the numerical value. From these estimates, supercurvature modes in small $\mathrm{CH}$ spaces $(\operatorname{Vol}(\mathcal{M}) \sim 1)$ are unlikely to be observed.

To compute the value of eigenfunctions inside the fundamental domain, one needs to solve Eq.(29). The singular decomposition method is the most suitable numerical method for solving any linear equation with a singular matrix $A$, which can be decomposed as

$$
A=U^{\dagger} D V
$$

where $U$ and $V$ are unitary matrices and $\mathrm{D}$ is a diagonal matrix. If $D_{i i}$ in $D$ is almost zero then the complex conjugate of the i-th row in $\mathrm{V}$ is an approximated solution of Eq.(29). The number of the "almost zero" diagonal elements in D is equal to the multiplicity number. Substituting the values of the eigenfunctions and their normal derivatives on the boundary into Eq.(10), the values of the eigenfunctions inside the fundamental domain can be computed. Eigenfunctions $k=5.41$ and

\footnotetext{
${ }^{5}$ The inradius $r_{-}$is the radius of the largest simply-connected sphere in the fundamental domain, and the outradius $r_{+}$is the radius of the smallest sphere that can enclose the fundamental domain. $r_{-}=0.535, r_{+}=0.7485$ for the Thurston manifold.
} 

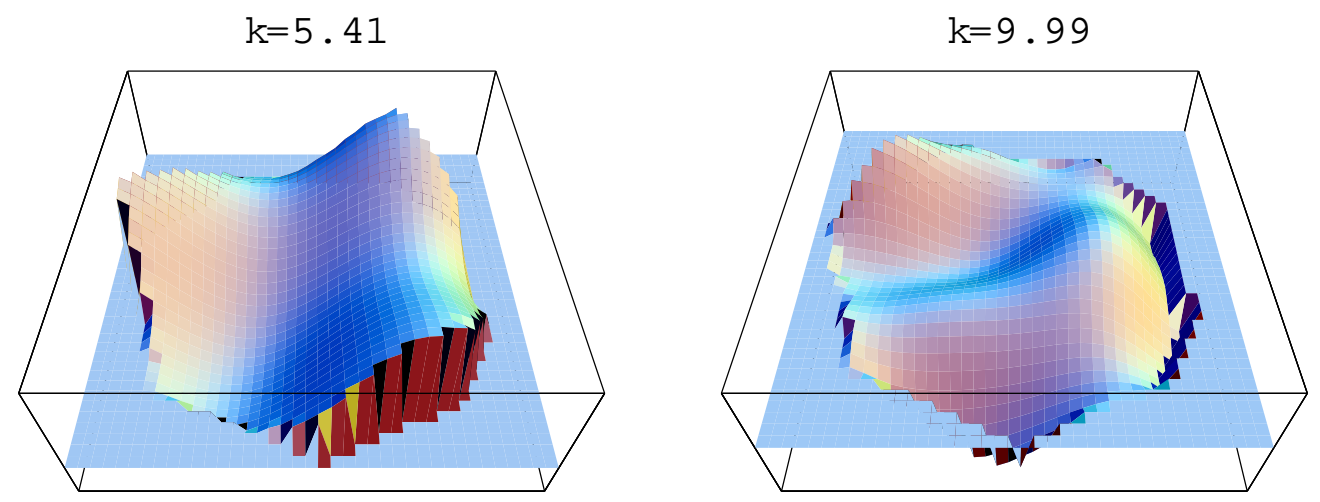

Figure 2: Eigenfunctions $u_{\nu}$ on a slice $x_{3}=0.0087$

$k=9.99$ in Poincaré coordinates plotted as $\left(x_{1}, x_{2}, h\right)$, where $h=u_{\nu}\left(x_{1}, x_{2}, 0.0087\right)$ are shown in figure 2. The eigenfuctions we computed are all real-valued. Note that the non-degenerated eigenfunctions must be real-valued.

The numerical accuracy of the obtained eigenvalues is roughly estimated as follows. First, let us write the obtained numerical solution in terms of the exact solution as $k=k_{0}+\delta k$ and $u_{k}(\mathbf{x})=u_{k_{0}}(\mathbf{x})+\delta u_{k}(\mathbf{x})$, where $k_{0}$ and $u_{k_{0}}(\mathbf{x})$ are the exact eigenvalue and eigenfunction, respectively. The singular decomposition method enables us to find the best approximated solution which satisfies

$$
[A]\{x\}=\epsilon, \quad|\epsilon|<<1,
$$

where $\epsilon$ is a $\mathrm{N}$-dimensional vector and || denotes the Euclidean norm. It is expected that the better approximation gives the smaller $|\epsilon|$. Then Eq. (33) can be written as,

$$
\int_{\Omega} G_{k_{0}+\delta k}\left(\mathbf{x}, \mathbf{y}_{J}\right)\left(\Delta+\left(k_{0}+\delta k\right)^{2}\right)\left(u_{k_{0}}(\mathbf{x})+\delta u_{k}(\mathbf{x})\right) \sqrt{g} d V_{\mathbf{x}}=\epsilon\left(\mathbf{y}_{J}\right) .
$$

Ignoring the terms in second order, Eq. (34) is reduced to

$$
\int_{\Omega} G_{k}\left(\mathbf{x}, \mathbf{y}_{J}\right)\left(\left(\Delta+k_{0}^{2}\right) \delta u_{k}(\mathbf{x})+2 k \delta k u_{k}(\mathbf{x})\right) \sqrt{g} d V_{\mathbf{x}}=\epsilon\left(\mathbf{y}_{J}\right) .
$$

Since it is not unlikely that $\left(\Delta+k_{0}^{2}\right) \delta u_{k}(\mathbf{x})$ is anticorrelated to $2 k \delta k u_{k}(\mathbf{x})$, we obtain the following relation by averaging over $\mathbf{y}_{J}$,

$$
2 k|\delta k|\left\langle\left|\int_{\Omega} G_{k}\left(\mathbf{x}, \mathbf{y}_{J}\right) u_{k}(\mathbf{x}) \sqrt{g} d V_{\mathbf{x}}\right|\right\rangle \sim<|\epsilon|>,
$$



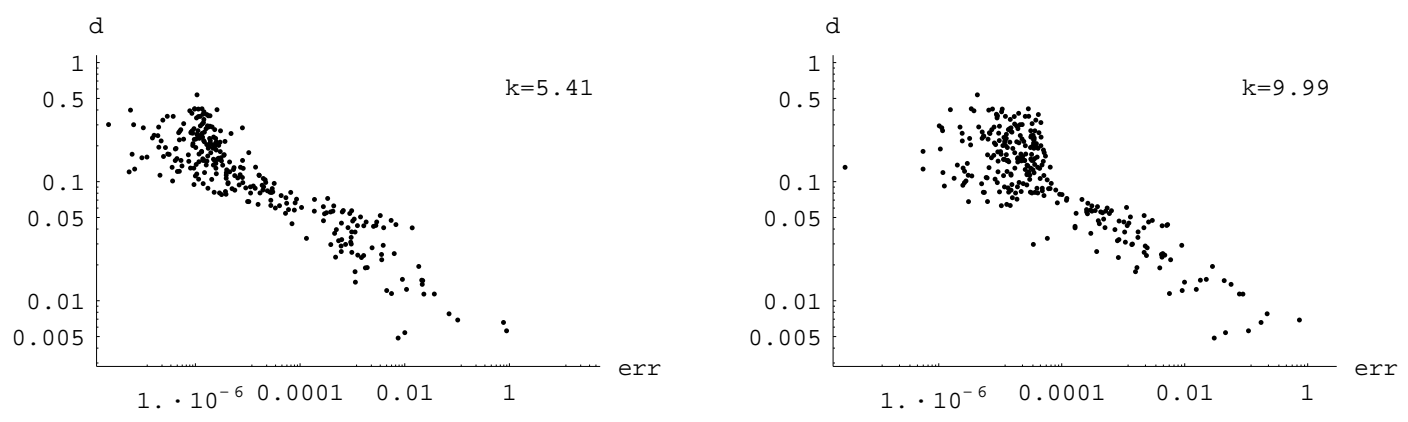

Figure 3: Error (err) versus hyperbolic distance to the boundary for 291 points inside the fundaental domain. $\mathrm{d}$ denotes the hyperbolic distance from the evaluation point $\mathbf{x}$ to the nearest point on the boundary.

where $<>$ denotes the averaging over $\mathbf{y}_{J}$ Thus one can estimate the expected deviation of the calculated eigenvalue $|\delta k|$ from $u_{k}(\mathbf{x})$ and $\epsilon\left(\mathbf{y}_{J}\right)$. We numerically find that $|\delta k|=0.005$ for $k=5.41$ and $|\delta k|=0.01$ for $k=9.91$. The other deviation values lie in between 0.005 and 0.01 .

By computing the second derivatives, one can also estimate the accuracy of the computed eigenfunctions. The accuracy parameter err is defined as

$$
\operatorname{err}(k, \mathbf{x}) \equiv\left(\Delta+k^{2}\right) u_{k}(\mathbf{x}),
$$

where $u_{k}(\mathbf{x})$ is normalized $\left(\mathcal{O}\left(u_{k}(\mathbf{x})\right) \sim 1\right)$. We see from figure 3 that the accuracy becomes worse as the evaluation point approaches the boundary. However, for points with hyperbolic distance $d>0.1$ between the evaluating point and the nearest boundary, the errors are very small indeed: err $\lesssim 10^{-4 \sim-5}$. This result is considered to be natural because the characteristic scale $L$ of the boundary elements is $\sim 0.07$ for our 1168 elements. If $d<L$, the integrands in Eq.(10) become appreciable on the neighborhood of the nearest boundary point because the free Green's function approximately diverges on the point. In this case, the effect of the deviation from the exact eigenfunction is significant. If $d>>L$, the integrand on all the boundary points contributes almost equally to the integration so that the local deviations are cancelled out.

As we shall see in the next section, expansion coefficients are calculated using the values of eigenfunctions on a sphere. Since the number of evaluating points 
which are very close to the boundary is negligible on the sphere, expansion coefficients can be computed with relatively high accuracy. 


\section{Statistical properties of eigenmodes}

Properties of eigenmodes of the Laplace-Beltrami operator are determined by the Helmholtz equation. Therefore, at first glance it does not seem to make a sense to study the statistical properties of the eigenmodes.

However, if one recognizes the Laplace-Beltrami operator in a $\mathrm{CH}$ space as the Hamiltonian in a quantum mechanical system, each eigenmode can be interpreted as a wavefunction in an eigenstate. Since the corresponding classical system is known to be a typical chaotic system (K-system), it is natural to assume that the imprints of classical chaos is hidden in the corresponding quantum system.

Recent studies have demonstrated that some of the statistical properties of energy spectrum are in accordance with the universal prediction of random-matrix theory(RMT) [20, 21]. RMT also predicts that the squared expansion coefficients $\left|a_{i}\right|^{2}$ of an eigenstate with respect to a generic basis are Gaussian distributed [9, 10, [1]. In the limit $N \rightarrow \infty, x=\left|a_{i}\right|^{2}$ obeys the statistics given for three universality classes of the orthogonal (GOE, $\mu=1$ ), unitary (GUE, $\mu=2$ ) and symplectic (GSE, $\mu=4$ ) ensembles, each distribution function $P$ is given by

$$
P_{\mu}(x)=\left(\frac{\mu}{2}\right)^{\mu / 2} \Gamma(\mu / 2) x^{\mu / 2-1} e^{-\mu x / 2} .
$$

In our case, as the time-reversal symmetry of the Hamiltonian implies, one expects that $\left|a_{i}\right|^{2}$ obeys the GOE prediction. In order to apply the GOE prediction to the statistical properties of eigenstates on $\mathrm{CH}$ spaces, one needs to find a set of orthonormal basis but no closed analytic expression is known for any $\mathrm{CH}$ spaces. To avoid the problem, Aurich and Steiner noticed that the wavefunctions on the hyperbolic octagons can be continued onto the universal covering space $\mathbb{H}^{2}$, and eigenstates can be expanded in terms of circular-waves [9]. They numerically found that the squared expansion coefficients obeys the GOE prediction in highly excited states of a hyperbolic asymmetrical octagon model.

We extend their method to three-dimensional $\mathrm{CH}$ models where we consider only low-lying modes. First, we normalize the obtained 14 eigenfunctions on the Thurston manifold. The eigenfunctions are naturally continued onto the whole unit Poincaré ball by the periodic boundary condition. As a "generic basis", we 
consider a set of orthonormal eigenfunctions $Q_{\nu l m}$ (T-complete functions) on the unit pseudosphere which is isometric to the Poincaré ball,

$$
\begin{aligned}
Q_{\nu l m} & \equiv X_{\nu l}(\chi) Y_{l m}(\theta, \phi) \\
X_{\nu l} & \equiv(-1)^{l+1} \sqrt{\frac{2}{\pi}}\left(\prod_{n=0}^{l}\left(n^{2}+\nu^{2}\right)\right)^{-1 / 2} \sinh ^{l} \chi \frac{d^{l+1}(\cos \nu \chi)}{d(\cosh \chi)^{l+1}} \\
& =\frac{\Gamma(l+1+\nu i)}{\Gamma(\nu i)} \sqrt{\frac{1}{\sinh \chi}} P_{\nu i-1 / 2}^{-l-1 / 2}(\cosh \chi), \quad \nu^{2}=k^{2}-1,
\end{aligned}
$$

where $P, Y_{l m}$ and $\Gamma$ denote the associated Legendre function, the spherical harmonics and gamma function, respectively. $P$ can be written in terms of the hypergeometric function ${ }_{2} \mathcal{F}_{1}$ 22],

$$
P_{\nu i-1 / 2}^{-l-1 / 2}(\cosh \chi)=\frac{\left(\operatorname{coth} \frac{1}{2} \chi\right)^{-l-1 / 2}}{\Gamma\left(\frac{3}{2}+l\right)}{ }_{2} \mathcal{F}_{1}\left(\frac{1}{2}-\nu i, \frac{1}{2}+\nu i ; \frac{3}{2}+l ;-\sinh ^{2} \frac{1}{2} \chi\right) .
$$

Eigenfunctions $u_{\nu}$ can be expanded in terms of $Q_{\nu l m}$ 's as

$$
u_{\nu}=\sum_{l m} \xi_{\nu l m} X_{\nu l}(\chi) Y_{l m}(\theta, \phi)
$$

Note that each $u_{\nu}$ has no components with $\nu^{\prime} \neq \nu$ because $Q_{\nu l m}$ 's are complete and linearly independent.

At first glance the computation of $\xi_{\nu l m}$ in Eq. (41) seems cumbersome as the domain of the integration extends over the whole pseudosphere. Fortunately, one can obtain $\xi_{\nu l m}$ by evaluating two-dimensional integrals. $\xi_{\nu l m}$ can be written as

$$
\xi_{\nu l m} X_{\nu l}\left(\chi_{o}\right)=\int u_{\nu}\left(\chi_{o}, \theta, \phi\right) Y_{l m}^{*}(\theta, \phi) d \Omega
$$

which is satisfied for the arbitrary value of $\chi_{o}$. In practice the numerical instability occurs in the region where the absolute value of $X_{\nu l}$ is too small. In our computation, the values of $\chi_{o}$ are chosen as shown in table 2. Thus $\xi_{\nu l m}$ can be computed if one obtains the values of eigenfunctions on the sphere

$$
x_{1}=\tanh \frac{\chi_{o}}{2} \sin \theta \cos \phi, x_{2}=\tanh \frac{\chi_{o}}{2} \sin \theta \sin \phi, x_{3}=\tanh \frac{\chi_{o}}{2} \cos \theta
$$

with radius $\chi_{o}$.

In order to compute the values of eigenfunctions on the sphere with radius longer 


\begin{tabular}{|c|c|c|c|}
\hline & $0 \leq 1<8$ & $8 \leq 1 \leq 13$ & $13<1 \leq 18$ \\
\hline $\mathrm{k}<8$ & 0.53 & 1.3 & 1.6 \\
$\mathrm{k}>8$ & 0.53 & 1.1 & 1.3 \\
\hline
\end{tabular}

Table 2: An example of choice of $\chi_{o}$ for which the absolute value of $X_{\nu l}\left(\chi_{o}\right)$ is not too small.

than the inradius $r_{-}=0.535$, the points outside of the fundamental domain must be pulled back to the inside, since Eq.(10) is valid only if $\mathbf{y}$ is a set of coordinates of an internal point.

The plots of eigenfunctions on a sphere $\chi_{o}=1.6$ are shown in figure 1 and figure 5. Apparent structure of the eigenfunctions on the sphere seems complicated. However, some regular patterns are hidden in the structure due to the periodic boundary conditions. Actually, there are pairs of highly correlated points on the sphere, since any partial surface $S_{i 1}$ of the sphere that is enclosed by copies of the boundary of the fundamental domain pulled back to inside the fundamental domain by the corresponding element of the discrete isometry group intersects another partial surface $S_{i 2}$ that is pulled back to inside the fundamental domain. To evaluate the correlation pattern, let us estimate how often a sphere with radius $\chi=\chi_{o}$ intersects the copies of the fundamental domain. The approximate number $n_{1}$ of the copies of the fundamental domain inside the sphere with radius (in proper length) $\chi_{o}$ is given by

$$
n_{1}=\frac{\pi\left(\sinh \left(2 \chi_{o}\right)-2 \chi_{o}\right)}{\operatorname{Vol}\left(Q_{2}\right)}
$$

From this formula, in the case of the Thurston manifold, $n_{1} \sim 29$ if $\chi_{o}=1.6$. Because the sphere intersects the fundamental domain at random, the copies of the fundamental domain on the sphere stick out their half portions on average. Therefore, the approximate number $n_{2}$ of the copies that intersect the sphere is given by

$$
n_{2}=\frac{\pi\left(\sinh \left(2\left(\chi_{o}+r_{\text {ave }}\right)\right)-\sinh \left(2\left(\chi_{o}-r_{\text {ave }}\right)\right)-4 r_{\text {ave }}\right)}{\operatorname{Vol}\left(Q_{2}\right)},
$$

where $r_{\text {ave }}=\left(r_{+}+r_{-}\right) / 2$. This estimate gives $n_{2} \sim 120$ if $\chi_{o}=1.6$. Approximating each eigenmode by de Broglie waves, we obtain the corresponding fluctuation scale $\delta A$ in steradian on the sphere,

$$
\delta A=\frac{4 \pi}{n_{2}} \frac{4 \pi^{2}}{k^{2}}
$$




$$
=\frac{16 \pi^{2} \operatorname{Vol}\left(Q_{2}\right)}{k^{2}\left(\sinh \left(2\left(\chi_{o}+r_{\text {ave }}\right)\right)-\sinh \left(2\left(\chi_{o}-r_{\text {ave }}\right)\right)-4 r_{\text {ave }}\right)} .
$$

Thus correlation patterns are observed in pairs of patches with typical size $\delta A$. When $\chi_{o}=1.6$, angular fluctuation scales are given as $\delta l \sim \sqrt{\delta A}=21^{\circ}, 12^{\circ}$, for $k=5.41,9.99$, respectively.

Next, we extract a set of independent variables from $\xi_{\nu l m}$ 's. In general, any $Q_{\nu l m}$ is related to $Q_{\nu l-m}$ as

$$
Q_{\nu l-m}=(-1)^{m} Q_{\nu l m}^{*} F(\nu, l)
$$

where

$$
F(\nu, l)=\frac{\Gamma(l+\nu i+1)}{\Gamma(\nu i)} \frac{\Gamma(-\nu i)}{\Gamma(l-\nu i+1)} .
$$

If $u_{\nu}$ is real, from Eq.(47),

$$
\begin{aligned}
u_{\nu} & =\sum_{l m} \xi_{\nu l m} Q_{\nu l m} \\
& =\sum_{l m} \xi_{\nu l m}^{*} Q_{\nu l m}^{*} \\
& =\sum_{l m}(-1)^{-m} \xi_{\nu l-m}^{*} Q_{\nu l m} F^{-1}(\nu, l),
\end{aligned}
$$

therefore,

$$
\xi_{\nu l m}=(-1)^{m} \xi_{\nu l-m}^{*} F^{-1}(\nu, l)
$$

Thus $\xi_{\nu l-m}$ can be written in terms of $\xi_{\nu l m}$. To extract a set of independent variables from $\xi_{\nu l m}$ 's, we rewrite Eq. (49) as follows

$$
\begin{aligned}
u_{\nu} & =\sum_{l, m \leq 0} \xi_{\nu l m} Q_{\nu l m}+\sum_{l, m>0} \xi_{\nu l m}^{*} Q_{\nu l m}^{*} \\
& =-\operatorname{Im}\left(\xi_{\nu 00}\right) \operatorname{Im}\left(Q_{\nu 00}\right)+\sum_{l>0} \sqrt{c_{\nu l}} \operatorname{Re}\left(\xi_{\nu l 0}\right) R_{\nu l 0} \\
& +\sum_{l>0, m>0} \sqrt{2}\left(\operatorname{Re}\left(\xi_{\nu l m}\right) \sqrt{2} \operatorname{Re}\left(Q_{\nu l m}\right)-\operatorname{Im}\left(\xi_{\nu l m}\right) \sqrt{2} \operatorname{Im}\left(Q_{\nu l m}\right)\right)
\end{aligned}
$$

where

$$
R_{\nu l 0}=\left(c_{\nu l}\right)^{-1 / 2}\left(\operatorname{Re}\left(Q_{\nu l 0}\right)+\frac{1-F(\nu, l)}{1+F(\nu, l)} \operatorname{Im}\left(Q_{\nu l 0}\right) i\right)
$$




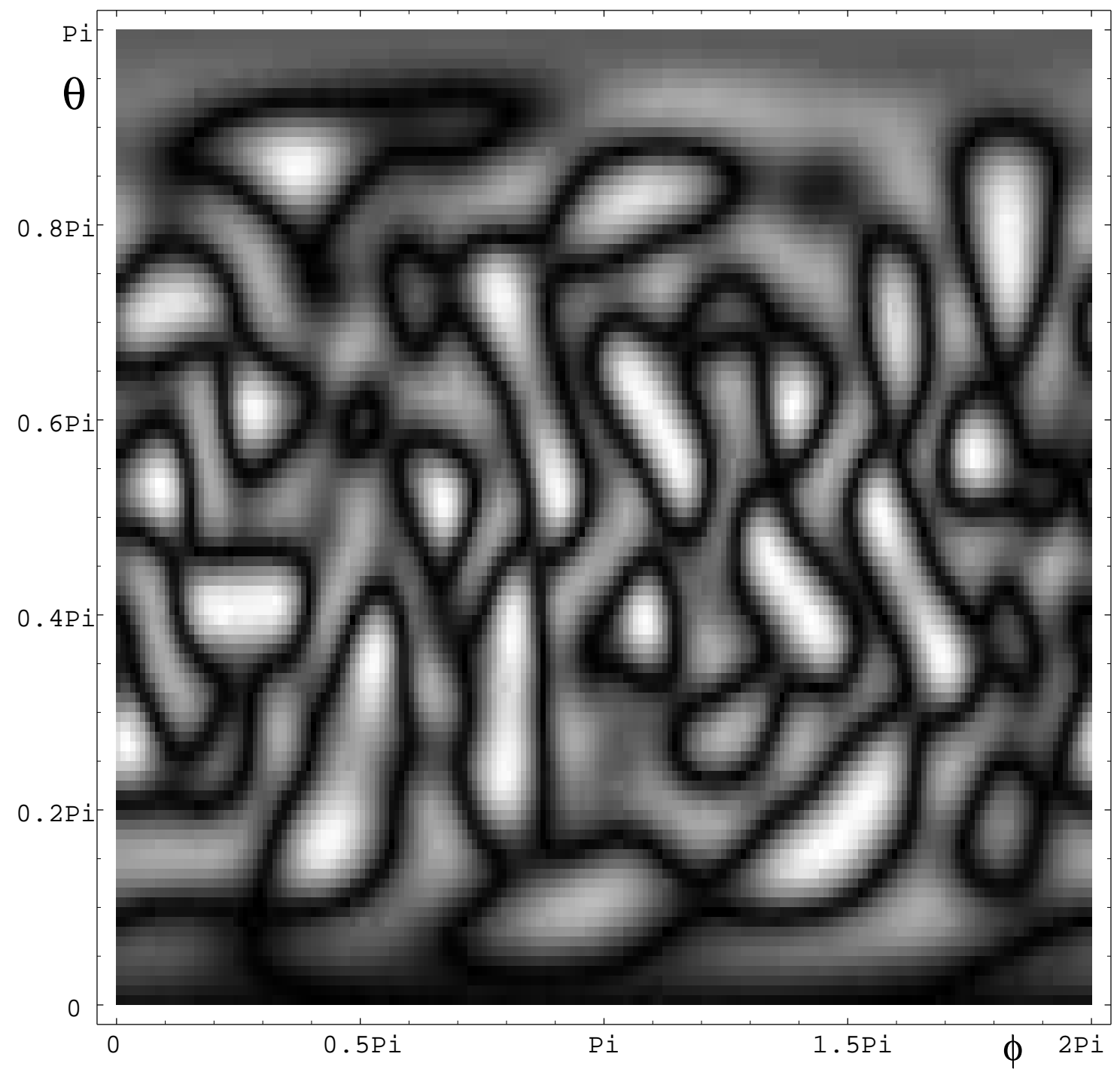

Figure 4: Absolute values of eigenfunction $u(k=5.41)$ on sphere $\chi_{o}=1.6$ 


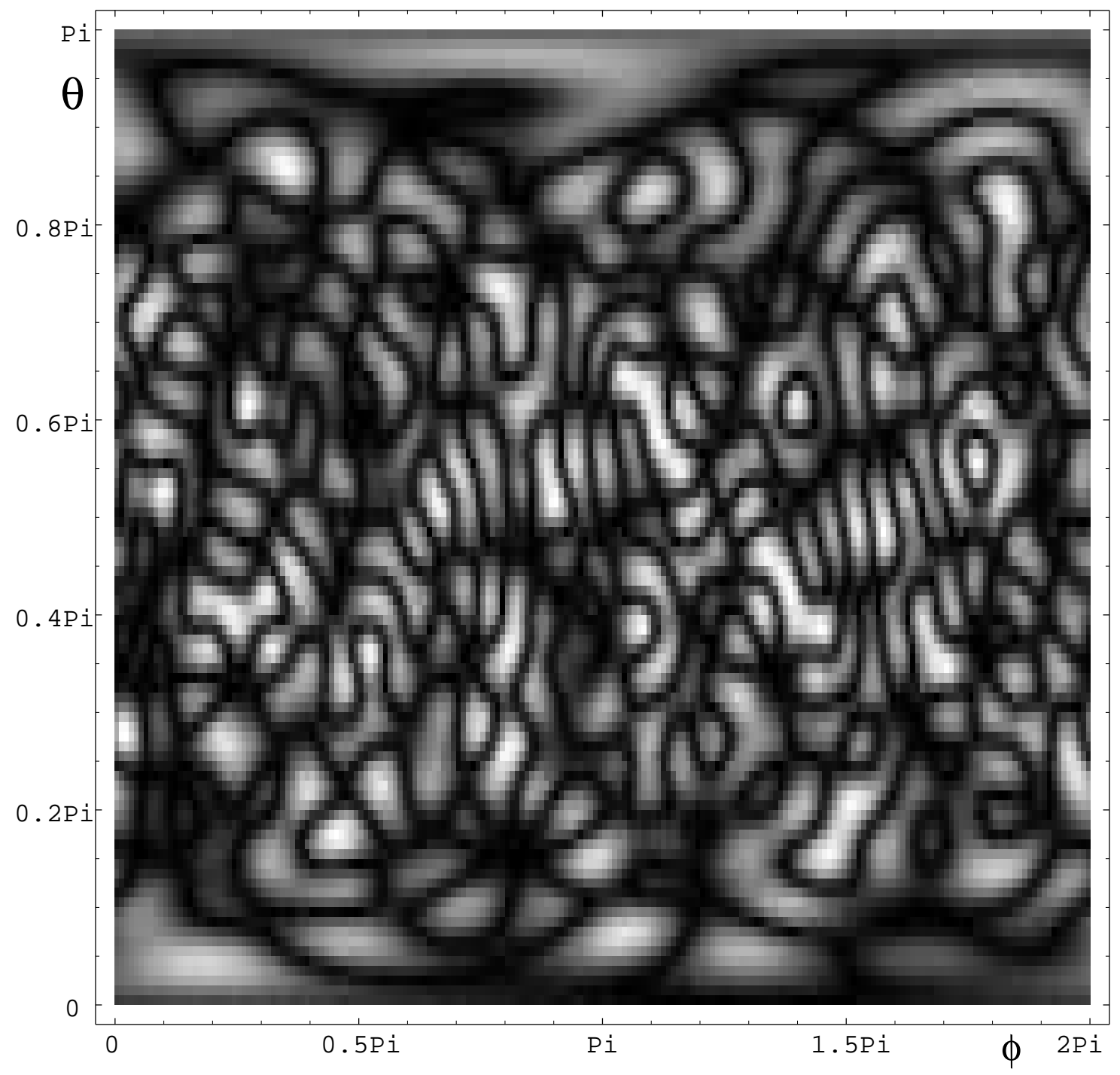

Figure 5: Absolute values of eigenfunction $u(k=9.99)$ on sphere $\chi_{o}=1.6$ 
and

$$
\begin{aligned}
c_{\nu l} & =1-\frac{(1-F(\nu, l))^{2}}{2 F(\nu, l)}-\left\{\left(\frac{1-F(\nu, l)}{1+F(\nu, l)}\right)^{2}+1\right\} \\
& \times \operatorname{Im}\left(\frac{\Gamma(l+\nu i+1)}{\Gamma(\nu i)}\right)^{2}\left|\frac{\Gamma(\nu i)}{\Gamma(l+\nu i+1)}\right|^{2} \\
& =\frac{2}{1+\operatorname{Re}(F(\nu, l))} .
\end{aligned}
$$

Thus the real eigenfuctions can be written in terms of real independent coefficients $a_{\nu l m}$ and real-valued $R_{\nu l m}$,

$$
u_{\nu}=\sum_{l, m} a_{\nu l m} R_{\nu l m}
$$

where

$$
\begin{array}{ll}
a_{\nu 00}=-\operatorname{Im}\left(\xi_{\nu 00}\right), \quad a_{\nu l 0}=\sqrt{c_{\nu l}} \operatorname{Re}\left(\xi_{\nu l 0}\right), \quad l>0, \\
a_{\nu l m}=\sqrt{2} \operatorname{Re}\left(\xi_{\nu l m}\right), \quad m>0, \quad a_{\nu l m}=-\sqrt{2} \operatorname{Im}\left(\xi_{\nu l-m}\right), \quad m<0,
\end{array}
$$

and

$$
\begin{aligned}
& R_{\nu 00}=\operatorname{Im}\left(Q_{\nu 00}\right), \\
& R_{\nu l m}=\sqrt{2} \operatorname{Re}\left(Q_{\nu l m}\right), \quad m>0, \quad R_{\nu l m}=\sqrt{2} \operatorname{Im}\left(Q_{\nu l-m}\right), \quad m<0 .
\end{aligned}
$$

Now we turn to the statistical properties of the coefficients $a_{\nu l m}$. As in [9], we consider the cumulative distribution of following quantities,

$$
\frac{\left|a_{\nu l m}-\bar{a}_{\nu}\right|^{2}}{\sigma_{\nu}^{2}}
$$

where $\bar{a}_{\nu}$ is the mean of $a_{\nu l m}$ 's and $\sigma_{\nu}^{2}$ is the variance. The cumulative distribution is compared to the cumulative RMT distribution functions which are directly derived from Eq.(38),

$$
\begin{aligned}
I_{\mu}(x) & =\int_{0}^{x} d x^{\prime} P_{\mu}\left(x^{\prime}\right) \\
& =\frac{\gamma(\mu / 2, \mu x / 2)}{\Gamma(\mu / 2)},
\end{aligned}
$$

where $\gamma(x, y)$ is the incomplete gamma function. To test the goodness of fit between the computed cumulative distribution function and that predicted by RMT, we use 
Kolmogorov-Smirnov statistic $D_{N}$ which is the least upper bound of all pointwise differences $\left|I_{N}(x)-I(x)\right|$ [23],

$$
D_{N} \equiv \sup _{x}\left|I_{N}(x)-I(x)\right|
$$

where $I_{N}(x)$ is the empirical cumulative distribution function defined by

$$
I_{N}(x)= \begin{cases}0, & x<y_{1}, \\ j / N, & y_{j} \leq x<y_{j+1}, \quad j=1,2, \ldots, N-1 \\ 1, & y_{j} \leq x\end{cases}
$$

where $y_{1}<y_{2}<\ldots<y_{N}$ are the computed values of a random sample which consists of $N$ elements. If $I_{N}(x)$ is "close" to $I(x)$, the observed $D_{N}$ must be so small that it falls within the range of possible fluctuations which depend on the size of the random sample. For the random variable $D_{N}$ for any $z>0$, it can be shown that the probability of $D_{N}<d$ is given by [24]

$$
\lim _{N \rightarrow \infty} P\left(D_{N}<d=z N^{-1 / 2}\right)=L(z)
$$

where

$$
L(z)=1-2 \sum_{j=1}^{\infty}(-1)^{j-1} e^{-2 j^{2} z^{2}} .
$$

From observed maximum difference $D_{N}=d$, we obtain the significant level $\alpha_{D}=$ $1-P$ which is equal to the probability of $D_{N}>d$. If $\alpha_{D}$ is found to be large enough, the hypothesis $I_{N}(x)=I(x)$ is verified. The computed cumulative distributions of $\left|a_{\nu l m}\right|^{2}$ and the $\operatorname{GOE}(\mu=1)$ prediction $I_{1}(x)$ for four examples are plotted in figure 6, and the maximum difference $d$ and the significant levels $\alpha_{D}$ for $0 \leq l \leq 10$ and $0 \leq l \leq 18$ are shown in table 3 . Note that the last digit in $\alpha_{N}$ is not guaranteed, since Eq.(61) is an asymptotic formula.

We see from figure 6 and table 3 that the agreement with GOE prediction is remarkably good. The Gaussian behavior for highly excited states is naturally expected as the semiclassical wavefunctions must reflect the chaotic nature in the corresponding classical systems. However, the Gaussian behavior for low-lying modes is not apparent at all. It is possible that the non-gaussianity behavior is rather prominent as these modes have fluctuations on large scale and that reflects 

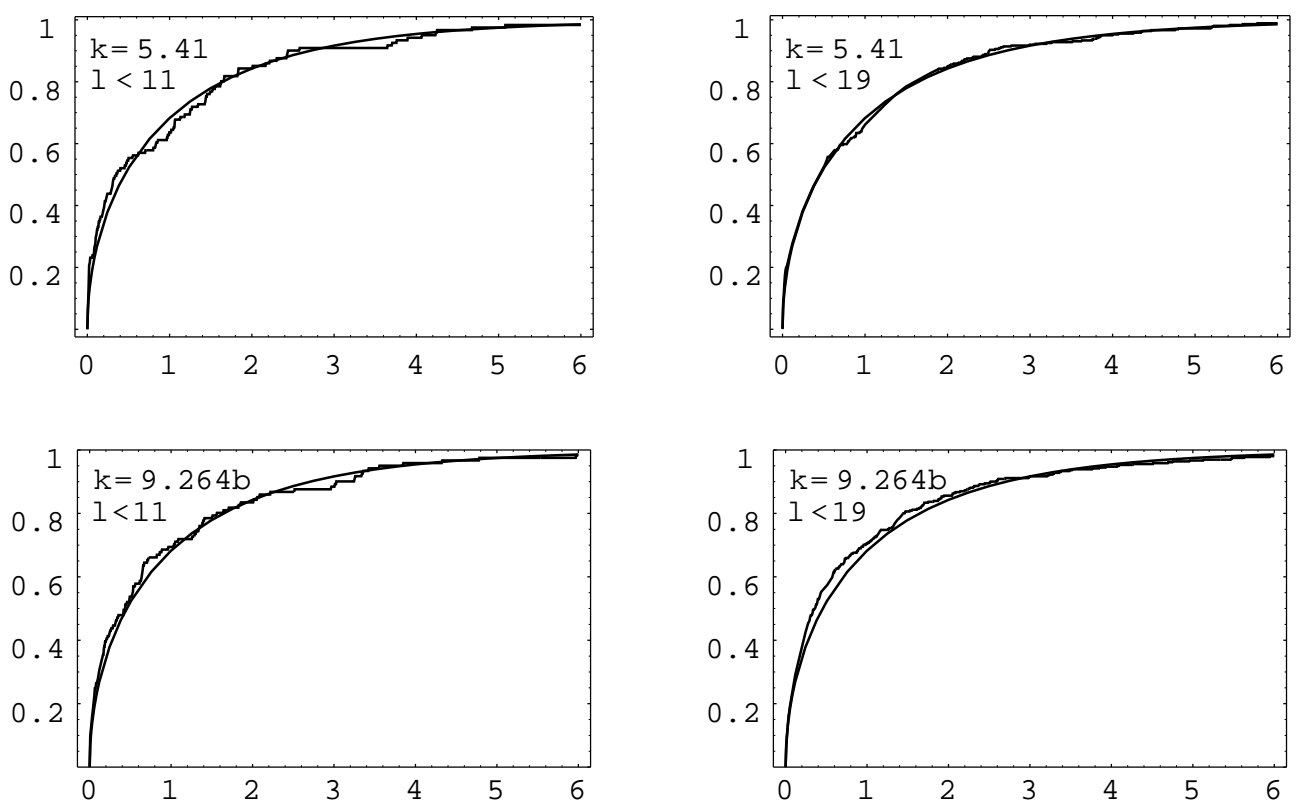

Figure 6: The cumulative distribution of $\left|a_{\nu l m}-\bar{a}_{\nu l m}\right|^{2} / \sigma^{2}$ are plotted with the theoretical curve for GOE prediction (full curve). The statistics are based on 121 expansion coefficients for $l<11$ and 361 coefficients for $l<19$.

the pure quantum mechanical behaviorf. Nevertheless, our numerical results serve to strengthen the hypothesis that the expansion coefficients behave as Gaussian pseudo-random numbers even for low-lying modes.

\footnotetext{
${ }^{6}$ The typical scale of angular fluctuation of $l$ mode is approximately $\pi /(2 l)$ and the typical scale of radial fluctuation of $k$ mode is approximately given by $2 \pi / k$.
} 


\begin{tabular}{c|cc|cc}
\hline & \multicolumn{2}{|c|}{$0 \leq l \leq 10$} & \multicolumn{2}{c}{$0 \leq l \leq 18$} \\
\hline $\mathrm{k}$ & $D_{N} \times 10^{2}$ & $\alpha_{D}$ & $D_{N} \times 10^{2}$ & $\alpha_{D}$ \\
\hline 5.41 & 9.42 & $23.4 \%$ & 4.29 & $51.8 \%$ \\
5.79 & 3.88 & $99.3 \%$ & 4.29 & $52.1 \%$ \\
6.81 & 5.95 & $78.6 \%$ & 3.47 & $77.8 \%$ \\
6.89 & 9.34 & $24.1 \%$ & 2.36 & $98.8 \%$ \\
7.12 & 7.12 & $57.2 \%$ & 2.59 & $96.8 \%$ \\
7.69 & 10.23 & $15.9 \%$ & 4.84 & $36.7 \%$ \\
8.30 & 6.38 & $70.8 \%$ & 3.88 & $65.0 \%$ \\
8.60 & 5.63 & $83.8 \%$ & 2.09 & $99.7 \%$ \\
8.73 & 9.46 & $22.9 \%$ & 2.78 & $94.3 \%$ \\
$9.26 \mathrm{a}$ & 7.21 & $55.6 \%$ & 3.46 & $78.1 \%$ \\
$9.26 \mathrm{~b}$ & 5.99 & $77.9 \%$ & 6.11 & $13.5 \%$ \\
9.76 & 7.41 & $52.0 \%$ & 4.57 & $43.8 \%$ \\
9.91 & 8.90 & $29.3 \%$ & 3.23 & $84.7 \%$ \\
9.99 & 4.27 & $98.0 \%$ & 3.72 & $70.0 \%$ \\
\hline ave. & 7.23 & $56.3 \%$ & 3.69 & $68.8 \%$ \\
\hline
\end{tabular}

Table 3: The Kolmogolov-Smirnov statistics $D_{N}$ and the significance levels $\alpha_{D}$ for the test of the hypothesis $I_{N}(x) \neq I(x)$ and their averages. $N=121$ for $0 \leq l \leq 10$ and $N=361$ for $0 \leq l \leq 18$. The mode $k=9.26$ is degenerated into two modes, which (after orthogonalization)are denoted by $k=9.26 a$ and $k=9.26 b$. 
Next we examine the randomness of $a_{\nu l m}$ 's. Because $a_{\nu l m}$ 's are actually determined by the Helmholtz equation, it is appropriate to describe $a_{\nu l m}$ 's as pseudorandom numbers. We apply the run test for testing randomness (see [23]).

Suppose that we have $n$ observations of the random variable $X$ and $\mathrm{m}$ observations of the random variable $Y$. The combination of those variables into $m+n$ observations placed in ascending order of magnitude yields

$\underline{x x x} \underline{y y} \underline{x x} \underline{y y y} \underline{x} \underline{y} \underline{x x} \underline{y y}$

where $x$ denotes an observation of $X$ and $y$ denotes an observation of $Y$. Each underlined group which consists of successive values of $X$ or $Y$ is called run. The statistics of number of runs are used for testing whether $X$ and $Y$ have the same the distribution function. Regardless of the type of the distribution function, the run number $r$ is known to behave as Gaussian random numbers in the limit $m, n \rightarrow \infty$.

The run test is also used as a test for randomness. Let $a_{1}, a_{2}, \ldots, a_{N}$ be the observed values of a random variable $A$. For simplicity, assume that $N$ is even. The median divides the observed values into a lower and an upper half. It is represented as $L$ if it falls below the median, and it is represented as $U$ if it falls above the median. For instance, a sequence

$\underline{U U U} \underline{L} \underline{U U} \underline{L L} \underline{U} \underline{U} \underline{L} \underline{U U}$,

has 8 numbers of runs $(r=8)$. The critical region for testing the hypothesis of randomness is of the form $r<c_{1}$ or $r>c_{2}$ where $c_{1}$ and $c_{2}$ is readily given by the Gaussian distribution function. The significant level $\alpha_{r}$ is the probability of $r>c_{1}$ or $r>c_{2}$. As the Kolmogolov-Smirnov test, $\alpha_{r}$ is given by the observed $r$.

The run numbers $r$ and the significant levels $\alpha_{r}$ are shown in table $₫$. High significant levels are again observed except for the one at $k=8.73$ for $0 \leq l \leq 18$. As the corresponding $r$ is larger than the averaged value, this may be due to the cyclic effect. On the whole, it is concluded that $a_{\nu l m}$ 's behave as if they are random variables. 


\begin{tabular}{c|cc|cc}
\hline & \multicolumn{2}{|c|}{$0 \leq l \leq 10$} & \multicolumn{2}{c}{$0 \leq l \leq 18$} \\
\hline $\mathrm{k}$ & $r$ & $\alpha_{r}$ & $r$ & $\alpha_{r}$ \\
\hline 5.41 & 62 & $85.5 \%$ & 185 & $67.3 \%$ \\
5.79 & 58 & $46.5 \%$ & 174 & $39.9 \%$ \\
6.81 & 69 & $14.4 \%$ & 196 & $11.4 \%$ \\
6.89 & 60 & $71.5 \%$ & 168 & $14.0 \%$ \\
7.12 & 69 & $14.4 \%$ & 184 & $75.2 \%$ \\
7.69 & 57 & $36.1 \%$ & 191 & $29.2 \%$ \\
8.30 & 63 & $71.5 \%$ & 177 & $59.8 \%$ \\
8.60 & 59 & $58.3 \%$ & 184 & $75.2 \%$ \\
8.73 & 70 & $10.0 \%$ & 201 & $3.5 \%$ \\
$9.26 \mathrm{a}$ & 56 & $27.3 \%$ & 177 & $59.8 \%$ \\
$9.26 \mathrm{~b}$ & 55 & $20.1 \%$ & 182 & $91.6 \%$ \\
9.76 & 59 & $58.3 \%$ & 182 & $91.6 \%$ \\
9.91 & 70 & $10.0 \%$ & 196 & $11.4 \%$ \\
9.99 & 58 & $46.5 \%$ & 179 & $75.2 \%$ \\
\hline ave. & 61.8 & $40.7 \%$ & 184 & $50.4 \%$ \\
\hline
\end{tabular}

Table 4: The run numbers $r$ and the significance levels $\alpha_{r}$ for the test of the hypothesis that $a_{\nu l m}$ 's are random variables. $N=121$ for $0 \leq l \leq 10$ and $N=361$ for $0 \leq l \leq 18$. The mode $k=9.26$ is degenerated into two modes, which (after orthogonalization)are denoted by $k=9.26 a$ and $k=9.26 b$. 


\section{Summary}

In this paper, we have demonstrated that the DBEM is eminently suitable for computing eigenmodes on $\mathrm{CH}$ spaces and we obtain some low-lying eigenmodes on a $\mathrm{CH}$ space called Thurston manifold which is the second smallest in the known $\mathrm{CH}$ manifolds and we have studied the statistical properties of these eigenmodes.

The low-lying eigenmodes are expanded in terms of eigenmodes on the pseudosphere, and we find that the expansion coefficients behave as if they are Gaussian random numbers. Why are they so random even for low-lying modes? It should be pointed out that the randomness of the expansion coefficients for low-lying eigenmodes is not the property of the eigenmodes themselves but rather the property of the images of eigenmodes on the whole universal covering space, since the fluctuation scales for low-lying eigenmodes are comparable to the the size of the fundamental domain. We conjecture that the origin of the random behavior of eigenmodes comes from the almost randomly distributed images of a set of points in the universal covering space.

Computation of eigenmodes is essential in simulating the CMB in $\mathrm{CH}$ cosmological models. As the DBEM needs only a set of face-to-face identification maps and the discretization of the corresponding fundamental domain, it can be applied to other $\mathrm{CH}$ spaces straightforwardly. However, the computation of the modes with small fluctuation scale $k \gg 1$ is still a difficult task as the number of modes increases as $N \propto k^{3}$.

Nevertheless, the contribution of the modes with small fluctuation to the temperature correlation of $\mathrm{CMB}$ can be estimated by assuming that the expansion coefficients for excited states $(k>>1)$ also behave as Gaussian pseudo-random numbers as well as that for low-lying modes. The assumption is numerically confirmed in a two-dimensional $\mathrm{CH}$ model [9].

If the observed Gaussian pseudo-randomness is found to be the universal behavior in $\mathrm{CH}$ spaces for low-lying modes as well as excited modes, the origin of the gaussianity of the CMB fluctuations can be partially explained. This is because the amplitude of the CMB fluctuation is written in terms of: 
1. expansion coefficients of the initial fluctuation in terms of eigenmodes on the $\mathrm{CH}$ space

2. expansion coefficients of eigenmodes on the $\mathrm{CH}$ space that are extended onto the whole pseudosphere in terms of eigenmodes on the pseudosphere.

\section{Acknowledgments}

I would like to thank my advisor Professor Kenji Tomita for his many helpful discussions and continuous encouragement. I would also like to thank Professor Toshiro Matsumoto for his extensive advice on the boundary element methods and Professor Jeff Weeks and the Geometry Center in University of Minnesota for providing me the data of $\mathrm{CH}$ spaces. Numerical computation in this work was carried out by HP Exemplar at the Yukawa Institute Computer Facility. I am supported by JSPS Research Fellowships for Young Scientists, and this work is supported partially by Grant-in-Aid for Scientific Research Fund (No.9809834).

\section{References}

[1] Stevens D, Scott D and Silk J 1993 Phys. Rev. Lett. 7120

[2] Oliveira-Costa A de, Smoot G F and Starobinsky A A 1996 Astrophys. J. 468457

[3] Levin J L, Barrow, J D, Bunn E F and Silk J 1997 Phys. Rev. Lett. 79974

[4] Levin J L, Scannapieco E and Silk J astro-ph/9802021

[5] Seriu M 1996 Phys. Rev. D 536902

[6] Aurich R and Steiner F 1989 Physica D 39169

[7] Grunewald F and Huntebrinker F 1996 Experimental Mathematics 557

[8] Cornish N J and Turok N G 1998 Class.Quant.Grav. 152699

[9] Aurich R and Steiner F 1993 Physica D 64185 
[10] Haake F and Zyczkowski K 1990 Phys. Rev. A 421013

[11] Brody T A, Flores J, French J B, Mello P A, Pandy A and Wong S S M 1981 Rev. Mod. Phys. 53385

[12] Brebbia C A and Dominguez J 1992 Boundary Elements - An introductory Course Second Edition (London: Computational Mechanics Publications)

[13] Tanaka M, Sladek V and Sladek J 1994 "Regularization techniques applied to boundary element methods" Applied Mechanics Reviews Vol.47 457

[14] Tabata M 1994 Bibunhouteishiki-no Suchikaihou II(Numerical Solution of Differential Equations II) (Tokyo: Iwanami Shoten Publishers)

[15] Johnson C 1987 Numerical Solution of Partial Differential Equations by the Finite Element Methods (Cambridge: Cambridge University Press)

[16] Elstrodt E, Grunewald F and Mennicke J 1983 Uspekhi Mat.Nauk 38 No.1 119

[17] Tomaschitz R 1989 Physica D 3442

[18] Telles J C F 1989 International Journal for Numerical Methods in Engineering 24959

[19] Hayami K and Brebbia C A 1987 Boundary Elements IX Vol.1 375

[20] Mehta M L 1991 Random Matrices (New York:Academic Press)

[21] Bohigas O 1991 "Random Matrix Theories and Chaotic Dynamics" in Proceedings of the 1989 Les Houches School on Chaos and Quantum Physics ed Giannoni A et al (Amsterdam: Elsevier)

[22] Harrison E R 1967 Rev. Mod. Phys. 39 No.4 862

[23] Hogg R V and Tanis E A 1977 Probability and Statistical Inference (New York: Macmillan Publishing Co., Inc.) 
[24] Birnbaum Z W 1962 Introduction to Probability and Mathematical Statistics (New York: Haper \& Brothers)

[25] Thurston W P 1979 The geometry and topology of three manifolds Princeton Lecture Notes (Now available via internet: http://www.msri.org/gt3m/)

[26] Thurston W P 1997 Three-Dimensional Geometry and Topology (Princeton University Press)

[27] Beardon A E 1983 The Geometry of Discrete Groups Graduate Texts in Mathematics 91 (New York: Springer-Verlag)

[28] Fomenko A T and Kunii T L 1997 Topological Modeling for Visualization (Tokyo: Springer-Verlag)

[29] Gabai D, Meyerhoff R and Thurston N 1996 Homotopy Hyperbolic 3Manifolds Are Hyperbolic MSRI preprint 1996-058 available at http://www.msri.org/

[30] Weeks J R 1998 SnapPea: A computer program for creating and studying hyperbolic 3-manifolds available at http://www.geom.umn.edu/software/download/snappea.htm]

[31] Weeks J R 1985 PhD thesis (Princeton: Princeton University)

[32] Matveev S V and Fomenko A T 1988 Russian Math Surveys 43 No.1 3

[33] Hodgson C and Weeks J 1994 Experimental Math. 261 


\section{A Boundary integral equation}

Here, we derive the boundary integral equation (11) in section 1. For simplicity, we prove the formula in 3-spaces.

First, we start with Eq.(10) with dimesnsion $M=3$. Although the integrand in Eq.(10) is divergent at $\mathbf{x}=\mathbf{y} \in \partial \Omega$, the integration can be regularized as follows. Let us draw a sphere with center $\mathbf{y} \in \partial \Omega$ with small radius $\epsilon$ and let $\Gamma_{\epsilon}$ be the outer spherical boundary and $\alpha$ and $\beta$ be the internal solid angle and external solid angle as shown in figure $\emptyset$,

$$
u(\mathbf{y})+\int_{\partial \Omega+\Gamma_{\epsilon}} G_{E}(\mathbf{x}, \mathbf{y}) \nabla_{i} u \sqrt{g} d S^{i}-\int_{\partial \Omega+\Gamma_{\epsilon}}\left(\nabla_{i} G_{E}(\mathbf{x}, \mathbf{y})\right) u \sqrt{g} d S^{i}=0 .
$$

The singular terms in Eq. 63) can be separated from non-singular terms as

$$
\begin{aligned}
\lim _{\epsilon \rightarrow 0} \int_{\partial \Gamma+\Gamma_{\epsilon}} G_{E}(\mathbf{x}, \mathbf{y}) \nabla_{i} u \sqrt{g} d S^{i} & =\int_{\partial \Gamma} G_{E}(\mathbf{x}, \mathbf{y}) \nabla_{i} u \sqrt{g} d S^{i} \\
& +\lim _{\epsilon \rightarrow 0} \int_{\Gamma_{\epsilon}} G_{E}(\mathbf{x}, \mathbf{y}) \nabla_{i} u \sqrt{g} d S^{i} \\
\lim _{\epsilon \rightarrow 0} \int_{\partial \Gamma+\Gamma_{\epsilon}}\left(\nabla_{i} G_{E}(\mathbf{x}, \mathbf{y})\right) u \sqrt{g} d S^{i} & =\int_{\partial \Gamma}\left(\nabla_{i} G_{E}(\mathbf{x}, \mathbf{y})\right) u \sqrt{g} d S^{i} \\
& +\lim _{\epsilon \rightarrow 0} \int_{\Gamma_{\epsilon}}\left(\nabla_{i} G_{E}(\mathbf{x}, \mathbf{y})\right) u \sqrt{g} d S^{i}
\end{aligned}
$$

If $\epsilon$ is sufficiently small, the region enclosed by $\Gamma_{\epsilon}$ can be approximated as an Euclidean subspace. In this region, the asymptotic form of the free Green's function $G_{E}$ takes the form

$$
\lim _{\mathbf{x} \rightarrow \mathbf{y}} G_{E}(\mathbf{x}, \mathbf{y})=-\frac{\exp (i k d)}{4 \pi d}=-\frac{1}{4 \pi d}-\frac{i k}{4 \pi}+\mathcal{O}(d),
$$

where $d$ is the Euclidean distance between $\mathbf{x}$ and $\mathbf{y}$. Taking the spherical coordinates $(\epsilon, \theta, \phi)$ with center $\mathbf{y}$, the singular terms in Eq.(64) are estimated as

$$
\begin{aligned}
\lim _{\epsilon \rightarrow 0} \int_{\Gamma_{\epsilon}} G_{E}(\mathbf{x}, \mathbf{y}) \nabla_{i} u(\mathbf{x}) \sqrt{g} d S^{i} & =\lim _{\epsilon \rightarrow 0}-\int_{\beta} \frac{1}{4 \pi \epsilon} \frac{\partial u(\mathbf{x})}{\partial n} \epsilon^{2} d \Omega=0 \\
\lim _{\epsilon \rightarrow 0} \int_{\Gamma_{\epsilon}}\left(\nabla_{i} G_{E}(\mathbf{x}, \mathbf{y})\right) u(\mathbf{x}) \sqrt{g} d S^{i} & =\lim _{\epsilon \rightarrow 0} \int_{\beta} \frac{1}{4 \pi \epsilon^{2}} \frac{\partial \epsilon(\mathbf{x})}{\partial n} u(\mathbf{x}) \epsilon^{2} d \Omega \\
& =\frac{\beta}{4 \pi} u(\mathbf{x}),
\end{aligned}
$$




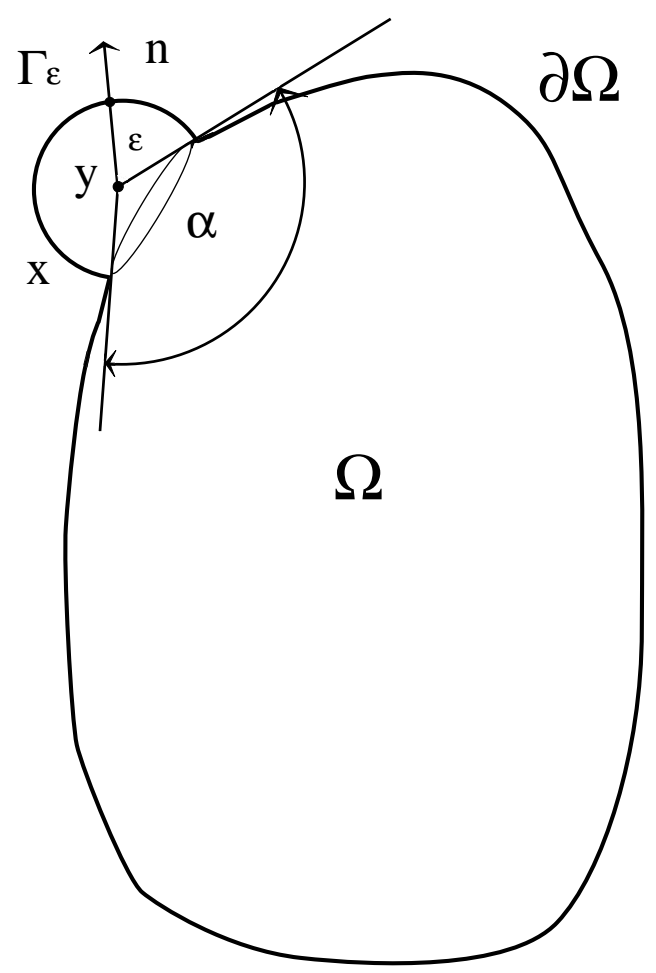

Figure 7: Boundary integral

where $d \Omega$ denotes the infinitesimal solid angle element. Taking the limit $\epsilon \rightarrow 0$ in Eq.(63), we have the boundary integral equation in 3-spaces,

$$
\frac{1}{4 \pi} \alpha(\mathbf{y}) u(\mathbf{y})+\int_{\partial \Omega} G_{E}(\mathbf{x}, \mathbf{y}) \nabla_{i} u \sqrt{g} d S^{i}-\int_{\partial \Omega}\left(\nabla_{i} G_{E}(\mathbf{x}, \mathbf{y})\right) u \sqrt{g} d S^{i}=0
$$

where $\alpha(\mathbf{y})$ denotes the internal solid angle at $\mathbf{y}$. If the boundary is smooth at $\mathbf{y}$, $\alpha(\mathbf{y})$ is equal to $2 \pi$ which gives the coefficients $1 / 2$ in Eq.(11). Similarly, one can prove the formula for $M=2$ and $M>3$. 


\section{B Three-dimensional hyperbolic spaces}

The discrete subgroup $\Gamma$ of $P S L(2, \mathbb{C})$ which is the orientation-preserving isometry group of the simply-connected hyperbolic 3-space $\mathbb{H}^{3}$ is called the Kleinian group. Any $\mathrm{CH}$ space (either manifold or orbifold) can be described as compact quotients $\mathcal{M}=\mathbb{H}^{3} / \Gamma$. The classification of the Kleinian group has not been completed. However, several procedures for constructing compact hyperbolic spaces are known. For further detail, see [25, 26, 27, 28].

The standard pseudospherical coordinates $(\chi, \theta, \phi)$ for $\mathbb{H}^{3}$ with curvature radius $R$ are given by

$$
\begin{aligned}
& X_{0}=R \cosh \chi, \quad X_{1}=R \sinh \chi \sin \theta \cos \phi \\
& X_{2}=R \sinh \chi \sin \theta \sin \phi, \quad X_{3}=R \sinh \chi \cos \theta
\end{aligned}
$$

with

$$
0 \leq \chi<\infty, \quad 0 \leq \theta<\pi, \quad 0 \leq \phi<2 \pi
$$

In these coordinates, the line element takes the form

$$
d s^{2}=\sum_{i=0}^{3}\left(d X_{i}\right)^{2}=R^{2}\left[d \chi^{2}+\sinh \chi^{2}\left(d \theta^{2}+\sin ^{2} \theta d \phi^{2}\right)\right] .
$$

The Poincaré representation is obtained by the transformation

$$
x_{1}=R \tanh \frac{\chi}{2} \sin \theta \cos \phi, \quad x_{2}=R \tanh \frac{\chi}{2} \sin \theta \sin \phi, \quad x_{3}=R \tanh \frac{\chi}{2} \cos \theta,
$$

which maps $\mathbb{H}^{3}$ into the open ball $\left\{\left(x_{1}, x_{2}, x_{3}\right) \in \mathbb{E}^{3} \mid x_{1}^{2}+x_{2}^{2}+x_{3}^{2}<R^{2}\right\}$ called the Poincaré ball. The line element in these coordinates takes the form

$$
d s^{2}=\frac{4\left(d x_{1}^{2}+d x_{2}^{2}+d x_{3}^{2}\right)}{\left(1-\frac{x_{1}^{2}+x_{2}^{2}+x_{3}^{2}}{R^{2}}\right)^{2}}
$$

and the geodesic distance $d$ between $\mathbf{x}$ and $\mathbf{x}^{\prime}$ is given by

$$
\cosh \left[R^{-1} d\left(\mathbf{x}, \mathbf{x}^{\prime}\right)\right]=1+\frac{2\left|\tilde{\mathbf{x}}-\tilde{\mathbf{x}}^{\prime}\right|^{2}}{\left(1-|\tilde{\mathbf{x}}|^{2}\right)\left(1-\left|\tilde{\mathbf{x}}^{\prime}\right|^{2}\right)}
$$


where || denotes the Euclidean norm and $\tilde{\mathbf{x}}=R^{-1} \mathbf{x}, \quad \tilde{\mathbf{x}}^{\prime}=R^{-1} \mathbf{x}^{\prime}$. Note that geodesics in the Poincaré ball are either diameters or semi-circles which orthogonally intersect with the boundary of the Poincaré ball.

In Poincaré coordinates, the metric is conformally flat so that the computation of the boundary integral equation becomes simpler.

Another commonly used set of coordinates is obtained from the upper-half space representation which is defined by the transformation

$$
\begin{aligned}
y_{1} & =\frac{\sinh \chi \sin \theta \cos \phi}{D}, y_{2}=\frac{\sinh \chi \sin \theta \sin \phi}{D}, y_{3}=\frac{1}{D}, \\
D & =\cosh \chi-\sinh \chi \cos \theta
\end{aligned}
$$

which maps $\mathbb{H}^{3}$ into the upper-half space $\mathbb{E}_{+}^{3}=\left\{\left(y_{1}, y_{2}, y_{3}\right) \in \mathbb{E}^{3} \mid y_{3}>0\right\}$. In these coordinates, the line element takes the form

$$
d s^{2}=\frac{R^{2}\left(d y_{1}^{2}+d y_{2}^{2}+d y_{3}^{2}\right)}{y_{3}^{2}} .
$$

The geodesic distance is given by

$$
\cosh \left[R^{-1} d\left(\mathbf{y}, \mathbf{y}^{\prime}\right)\right]=1+\frac{\left|\mathbf{y}-\mathbf{y}^{\prime}\right|^{2}}{2 y_{3} y_{3}^{\prime}} .
$$

In the upper-half space model, geodesics are either straight vertical lines or semicircles orthogonal to the boundary of the upper-half space. In this coordinates the metric is conformally flat as in Poincaré coordinates. If we represent a point $p$ on the upper-half space, as a quaternion whose fourth component equals zero [27, then the actions of $\operatorname{PSL}(2, \mathbb{C})$ on $\mathbb{H}^{3} \cup \mathbb{C} \cup\{\infty\}$ can be described by simple formulas,

$$
\tilde{\gamma}: p \rightarrow p^{\prime}=\frac{a p+b}{c p+d}, \quad a d-b c=1, \quad p \equiv z+y_{3} \mathbf{j}, \quad z=y_{1}+y_{2} \mathbf{i},
$$

where $\mathrm{a}, \mathrm{b}, \mathrm{c}$ and $\mathrm{d}$ are complex numbers and $1, \mathbf{i}$ and $\mathbf{j}$ are represented by matrices as,

$$
1=\left(\begin{array}{ll}
1 & 0 \\
0 & 1
\end{array}\right), \quad \mathbf{i}=\left(\begin{array}{cc}
i & 0 \\
0 & -i
\end{array}\right), \quad \mathbf{j}=\left(\begin{array}{cc}
0 & 1 \\
-1 & 0
\end{array}\right) .
$$

As $\bar{p}=z-y_{3} \mathbf{j}$, the action $\tilde{\gamma}$ is explicitly written as

$$
\begin{aligned}
& \tilde{\gamma}: \mathbb{H}^{3} \cup \mathbb{C} \cup\{\infty\} \rightarrow \mathbb{H}^{3} \cup \mathbb{C} \cup\{\infty\} \\
& \tilde{\gamma}:\left(z\left(y_{1}, y_{2}\right), y_{3}\right) \rightarrow\left(\frac{(a z+b)(\overline{c z+d})+a \bar{c} y_{3}^{2}}{|c z+d|^{2}+|c|^{2} y_{3}^{2}}, \frac{y_{3}}{|c z+d|^{2}+|c|^{2} y_{3}^{2}}\right)
\end{aligned}
$$


If we restrict the action $\tilde{\gamma}$ on $\mathbb{C} \cup\{\infty\}$, or equivalently, $y_{3}=0$, the action is described as

$$
\gamma: z \rightarrow z^{\prime}=\frac{a z+b}{c z+d}, \quad a d-b c=1, \quad z \equiv y_{1}+i y_{2}
$$

$\gamma$ is called the Möbius transformation, and $\tilde{\gamma}$ is called the extended Möbius transformation.

In the Klein (projective) model, the geodesics and planes are mapped into their Euclidean counterparts. Since the fundamental domain is enclosed by Euclidean planes in the Klein coordinates, the task of generating meshes is much easier than other coordinates. The transformation

$$
z_{1}=R \tanh \chi \sin \theta \cos \phi, \quad z_{2}=R \tanh \chi \sin \theta \sin \phi, \quad z_{3}=R \tanh \chi \cos \theta
$$

can be understood as the projection of the hyperboloid $\left(X_{0}, X_{1}, X_{2}, X_{3}\right)$ onto the interior of the sphere $\left(R, z_{1}, z_{2}, z_{3}\right)$ along lines originating from the origin $(0,0,0,0)$. The geodesic distance can be represented as

$$
\cosh \left[R^{-1} d\left(\mathbf{z}, \mathbf{z}^{\prime}\right)\right]=\frac{1-\tilde{\mathbf{z}} \cdot \tilde{\mathbf{z}}^{\prime}}{\sqrt{\left(1-|\tilde{\mathbf{z}}|^{2}\right)\left(1-\left|\tilde{\mathbf{z}}^{\prime}\right|^{2}\right)}} .
$$

where $\cdot$ denotes the Euclidean inner product and $\tilde{\mathbf{z}}=R^{-1} \mathbf{z}, \quad \tilde{\mathbf{z}}^{\prime}=R^{-1} \mathbf{z}^{\prime}$.

The possible values for the volume of the $\mathrm{CH}$ manifolds are bounded below and no upper bound exists. The minimal value has not yet been known, although Gabai et al have proved that $\operatorname{Vol}_{\min }>0.16668 \ldots R^{3}[29$. Thurston proposed a manifold $Q_{2}$ as a candidate for the three-dimensional hyperbolic manifold of the minimum volume $\operatorname{Vol}\left(Q_{2}\right)=0.98139 R^{3}$ [30. However, Weeks [31] and independently, Matveev and Fomenko [32] discovered a $\mathrm{CH}$ manifold $Q_{1}$ with the smallest value $\operatorname{Vol}\left(Q_{1}\right)=0.94272 R^{3}$ in the known $\mathrm{CH}$ manifolds and it is conjectured to be the one with the minimum volume. A computer program "SnapPea" by Jeff Weeks 33. has made it possible to catalog and study a large number of $\mathrm{CH}$ and non- $\mathrm{CH}$ spaces which include $Q_{1}, Q_{2}$ and thousands of cusped and non-cusped hyperbolic 3-manifolds. 

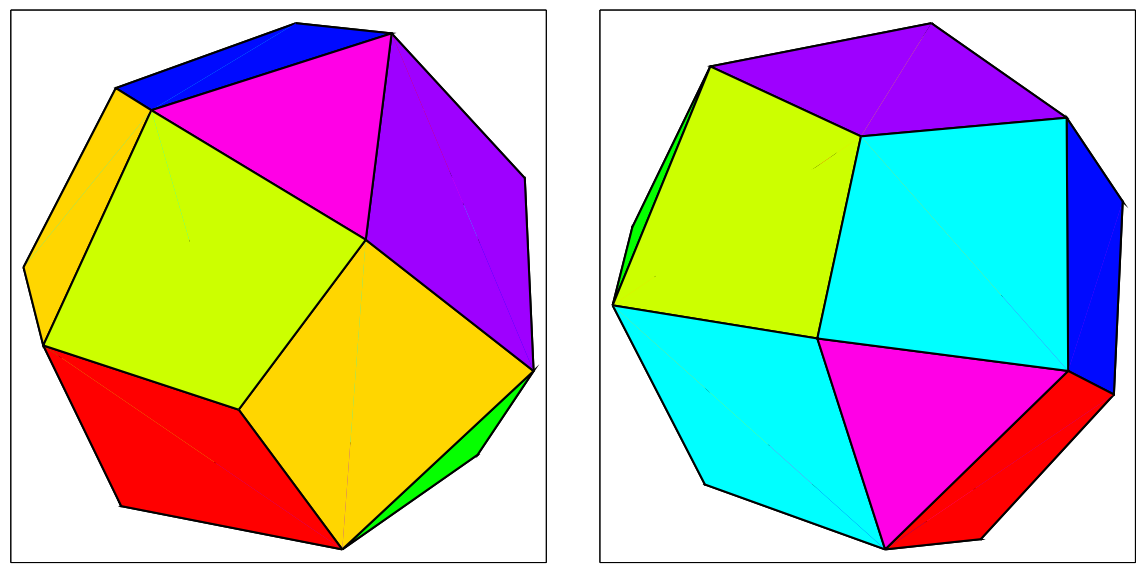

Figure 8: Fundamental domain of the Thurston manifold which is viewed from a point $(0,0,-1000)$ and a point $(0,0,1000)$ in the Klein coordinates, respectively.

Let us see how CH manifolds are characterized in the SnapPea. Any element of the discrete isometry group $\Gamma$ which is equivalent to the fundamental group $\pi_{1}(\mathcal{M})$ can be described as a word which is a product of generators $\left\{g_{1}, \ldots, g_{s}\right\}$,

$$
g=g_{m_{1}}^{n_{1}} \ldots g_{m_{j}}^{n_{j}}, \quad\left(j, n_{j} \in Z, \quad m_{j}=1, \ldots, s\right), \quad g \in \Gamma
$$

The above expression is not unique, since they are subject to a set of relations, each of which takes the form,

$$
\mathcal{I}=g_{l_{1}}^{k_{1}} \ldots g_{l_{j}}^{k_{j}}, \quad\left(j, k_{j} \in Z, \quad l_{j}=1, \ldots, s\right)
$$

where $\mathcal{I}$ denotes the identity. Note that different expression of $g$ is possible by choosing different generators. In the case of Thurston's manifold $Q_{2}, \Gamma$ has a simple presentation,

$$
\Gamma=\left\{a, b: a b^{3} a b a^{-2} b, \quad a b^{-1} a^{-1} b a b a^{-1} b^{-1} a b^{-1}\right\}
$$

where $a$ and $b$ are generators and words in the parenthesis are equal to identities. This representation is simple for describing $\Gamma$ but not convenient for describing the fundamental domain. Choosing a coordinate system centered at a point of locally maximum of the injectivity radius], generators which define the face identification

\footnotetext{
${ }^{7}$ The injectivity radius of a point $p$ is equal to half the length of the shortest periodic geodesic on $p$.
} 
maps in the pseudospherical coordinates can be described by 8 matrices (see appendix C), which implies that the number of the faces on the boundary of the fundamental domain is sixteen. For instance, the center $\left(X_{0}, X_{1}, X_{2}, X_{3}\right)=(1,0,0,0)$ is moved to a point $(1.63032,-0.5657,0.993147,-0.592943)$ by $T_{1}$. The fundamental domain of the Thurston manifold can be computed from these 8 matrices. First, let us make 16 copies of the basepoint $(1,0,0,0)$ that are obtained by multiplying the basepoint by 8 matrices and their inverse matrices. Next, the basepoint and the 16 copies are connected with 16 geodesic segments. If one put planes on the equidistant points on the segments at right angle, then one obtains the fundamental domain enclosed by the 16 planes. The face identifications are shown in figure 8, in which each color of the faces corresponds to one of the identification maps. For instance, a point on the face with red color in the left figure is identified with the corresponding point on the face with the same color in the right figure by $T_{1}$. 


\section{Table of matrices}

In the Minkowski coordinates $(t, x, y, z)$, the 8 generators which define the fundamental domain of the Thurston manifold with the basepoint $(1,0,0,0)$ are described by the following 8 matrices,

$$
\begin{aligned}
& T_{1}=\left(\begin{array}{cccc}
1.630319018827 & -0.823154893134 & 0.949658853916 & -0.280185985083 \\
-0.565700099811 & 0.926953540114 & 0.03235859132 & 0.678031458683 \\
0.993147485366 & -0.896311100897 & 1.03061185404 & 0.34757379707 \\
-0.592943144435 & -0.122341828367 & -0.915775117974 & 0.705669882356
\end{array}\right), \\
& T_{2}=\left(\begin{array}{cccc}
1.630319018827 & -0.404520784012 & -0.353128575811 & -1.170300494487 \\
-0.725744142114 & 1.032680902406 & 0.430856766432 & 0.524058355949 \\
-0.749088496222 & 0.153460453167 & -0.490988659119 & 1.138645511607 \\
0.755050970573 & 0.271398430022 & -0.835459379318 & -0.893561685534
\end{array}\right), \\
& T_{3}=\left(\begin{array}{cccc}
1.630319018827 & 0.687449077 & -0.510375368061 & -0.961702060597 \\
-0.493618131693 & 0.144914737528 & -0.255278318146 & 1.075867816726 \\
0.044016981085 & 0.704808911001 & 0.708779194553 & 0.053046648969 \\
-1.188420695119 & -0.977154210597 & 0.832435012465 & 0.874394274525
\end{array}\right), \\
& T_{4}=\left(\begin{array}{cccc}
1.630319018827 & -0.076636501663 & -1.186821280266 & -0.493479683947 \\
0.990384655015 & 0.188025082259 & -1.393877403859 & 0.051128429897 \\
-0.430529153566 & 0.973085065179 & 0.444575008386 & 0.202024429006 \\
-0.701229624437 & -0.153704839725 & 0.517690637565 & 1.09548811596
\end{array}\right), \\
& T_{5}=\left(\begin{array}{cccc}
1.630319018827 & -0.422808564099 & -0.669242281726 & 1.015523406733 \\
-1.169223329346 & 0.245239342961 & 1.092969619938 & -1.054683966146 \\
-0.48230025187 & 1.00284583704 & -0.146468308544 & -0.453277833869 \\
-0.241336645178 & -0.336043112815 & -0.481507711397 & -0.84467077995
\end{array}\right) \text {, } \\
& T_{6}=\left(\begin{array}{cccc}
1.630319018827 & -1.258320677027 & -0.085154694975 & -0.259456845811 \\
-0.334091294985 & 0.339168543325 & 0.989853092692 & 0.129508870322 \\
-1.230309911716 & 1.513335320951 & -0.161421439076 & 0.444321735047 \\
-0.180722524595 & 0.422080361796 & 0.03721956436 & -0.92364684978
\end{array}\right) \text {, }
\end{aligned}
$$




$$
\begin{gathered}
T_{7}=\left(\begin{array}{cccc}
1.815210205228 & -1.011761093044 & -1.117355434627 & 0.15114368155 \\
-1.394305392881 & 1.36137877709 & 0.985732513048 & -0.345060526033 \\
0.587810577053 & 0.152666848297 & -1.125213789716 & -0.236871347696 \\
0.073343616291 & -0.383407313596 & 0.103481918443 & -0.920689747139
\end{array}\right), \\
T_{8}=\left(\begin{array}{cccc}
1.815210205228 & -0.288909259155 & 0.774531377414 & -1.269496228642 \\
0.093635040681 & 0.602143185543 & -0.536756936203 & -0.598400448183 \\
-0.930634634266 & 0.833203272177 & -0.104192832263 & 1.077495699961 \\
-1.191696163383 & -0.163292533092 & -1.1405852444 & 1.045246666925
\end{array}\right) .
\end{gathered}
$$

